\title{
DOCUMENTS
}

Helmut Hirsch

Hans Pelger

\section{EIN UNVERÖFFENTLICHTER BRIEF VON KARL MARX AN SOPHIE VON HATZFELDT - ZUM STREIT MIT KARL BLIND NACH FERDINAND LASSALLES TOD}

Der hier vorgelegte Briefwechsel entstand vom 12. September bis 24. November 1864, in einer Zeit, die für Karl Marx ereignisreich war. Vom Tode Ferdinand Lassalles überrascht und ergriffen, reagierte er anders als beispielsweise Ferdinand Freiligrath, dessen Telegramm auf der Genfer Totenfeier am 2. September verlesen wurde. Marx schrieb am 12. September einen privat gehaltenen Kondolenzbrief $(\mathrm{Nr} 1)$ an Lassalles Lebensgefährtin Sophie von Hatzfeldt. Diese hatte eine große Aktivität entfaltet, um Lassalles Tod zu rächen, sein Ansehen zu ehren und sein Werk fortzusetzen. Dabei gedachte sie Marx, den sie seit 1847 kannte und gegen den sie im Grunde erhebliche Vorbehalte hatte, für ihre Zwecke einzusetzen, ohne ihn zuviel Einfluß erlangen zu lassen $(\mathrm{Nr} 2)$. Eine Zeitlang setzte sie ihre Hoffnung besonders darauf, Marx' Hilfe bei der Redaktion einer Broschüre über Lassalles unglückliches Ende zu erhalten. Diese Broschüre sollte eine „Apotheose”, ein „Racheschrei” und eine „Parteischrift” werden. Marx, der wie Friedrich Engels den Personenkult in der Arbeiterbewegung verachtete - beide hatten sich von Lassalle wegen seines politischen Auftretens als „Arbeiterdiktator" zurückgezogen -, sagte diplomatisch ab. Er stand der Gräfin aber bei der Gestaltung einer menschlich verständnisvoll zu verfassenden Lassalle-Broschüre beratend zur Seite.

Als Karl Blind - ein ehemaliger Parteigänger von Marx, der inzwischen nur noch republikanische Positionen vertrat und in selbstgefälliger Betriebsamkeit international agierte - in der deutschen, englischen und amerikanischen Presse mit einem „Republikanischen Protest” gegen den toten Lassalle auftrat und ihn des Arbeiterverrats bezichtigte, wandte sich die Gräfin am 21. November wiederum an Marx ( $\mathrm{Nr} 3$ ). Sie wußte von dessen Feindschaft zu Blind seit der Vogt-Affäre und verlangte von Marx die literarische „Vernichtung” des „Wichtigtuers”. Marx, der Blinds politische Machinationen bei der Erzeugung einer ,falschen öffentlichen Meinung" - zuletzt bei dem spektakulären Empfang Garibaldis in 
London - genau verfolgt hatte, zögerte zunächst (Nr 4). Er wollte nicht in den Verdacht kommen, Lassalles Unglück benutzt zu haben, um seine eigene alte Rechnung mit Blind zu begleichen. Als Blind jedoch erklären ließ, die Vogt-Affäre sei ,,abgetan”, obwohl er gegen Marx' veröffentlichte Anschuldigungen nichts anderes unternommen hatte, als „Stillschweigen” zu bewahren, griff Marx zur Feder. Hinzu kam, daß Joseph Weydemeyer von Amerika aus Blinds Protest in einem Brief an Engels als bedeutungslos beurteilt hatte.

Lassalle, der schon 1850 Bedenken gegen Blind geäußert hatte, war zu Anfang seiner Agitation im Juni 1863 von Blind mit einem „Freundeswort" angegriffen worden, das die Notwendigkeit einer selbständigen Arbeiterbewegung bestritt. Lassalle antwortete nicht öffentlich, es bestand aber wohl der im Einverständnis mit Marx gefaßte Plan, daß Ernst Dronke in Zusammenarbeit mit Wilhelm Eichhoff und unter Hinzuziehung von Bernhard Becker anklagendes Material über Blind sammeln und Lassalle für eine Abfertigung ,à la Julian Schmidt" zur Verfügung stellen sollte. Nach Lassalles plötzlichem Tod trat Marx aus „alter Freundschaft” dann selbst noch einmal öffentlich für Lassalle gegen Blind auf, ohne sich allerdings mit Lassalles ADAV-Agitation zu befassen. Er zahlte dem „grotesken Mazzini-Scapin" stellvertretend für alle, die über den ehemals gefürchteten Arbeiterführer hergefallen waren, seine „feige Frechheit” heim.

Besonders hervorzuheben ist, daß Marx in seinem Brief an die Gräfin die „Enthüllung” von Blind als einen „Grund” für seinen Eintritt in die Internationale Arbeiterassoziation bezeichnet. In der international bewegten Situation vom Herbst 1864 gab er nämlich seine seit langem praktizierte politische Zurückhaltung auf und wirkte als Repräsentant deutscher Arbeiter mit den wiederauflebenden englischen und französischen Arbeiterbewegungen zusammen. Schon bei der Gründung der IAA und der Abfassung ihrer programmatischen Dokumente machte er sich zur Aufgabe, die die Arbeiterbewegungen desorientierenden, klassenübergreifenden Republikanismus- und Nationalismus-Parolen von Mazzini, Ledru-Rollin und anderen ehemaligen „Demokratenkönigen”, und damit auch von Blind, zu bekämpfen.

Zum Aufbau der Dokumentation ist zu sagen, daß der Briefwechsel zwischen der Gräfin und Marx aus der Zeit vom 12. September bis 24. November chronologisch vorgelegt wird. Nur Marx’ Brieffragment vom 16. Oktober, das keine zusätzlichen Erkenntnisse über die Beziehung der beiden Briefpartner sowie deren jeweilige Handlungen bringt und dessen Uberlieferung noch genau zu erforschen bleibt, wurde ausgeklammert. Um die vielschichtigen Texte angemessen erläutern zu können, ist nur 
diese summarische, dem Verständnis der Zusammenhänge dienende Einführung vorangestellt; ausführliche Quellennachweise und Erläuterungen finden sich in den Anmerkungen.

Die Existenz des hier erstmals veröffentlichten zwölfseitigen MarxBriefes ist in der Forschung aus Erwähnungen im Briefwechsel von Marx und Engels bekannt gewesen. Anfang der 1960er Jahre gelangte eine Kopie der Originalhandschrift mit anderen Hatzfeldt-Materialien dank des Entgegenkommens von Ursula Gräfin von Hatzfeldt-Wildenburg und von Gerhard Ebel in das IISG, Amsterdam. Siegfried Bahne bereitete schon 1966 eine Veröffentlichung vor, die aber nicht realisiert werden konnte. Anfang dieses Jahres gab der Eigentümer, Hermann Graf Hatzfeldt, uns den Brief zur Veröffentlichung frei und legte die MarxOriginale ( $\mathrm{Nr} 1$ und 4) zur Autopsie vor. Dafür sprechen wir unseren besonderen Dank aus. Außerdem danken wir allen Personen und Institutionen, die in irgendeiner Weise unsere Veröffentlichung unterstützt haben.

Marx' Briefe werden nach den Originalhandschriften ohne Variantenverzeichnis wiedergegeben. Ein- oder zweifache Unterstreichungen erscheinen im Druck kursiv bzw. kursiv und unterstrichen. Die eckigen Klammern im Text entsprechen grundsätzlich dem Original. Marx' Paginierung ist aufgenommen und durch Zahlen in eckigen Klammern komplettiert. Die Briefe der Gräfin werden nach den Kopien der Originalhandschriften, ebenfalls ohne Varianten, abgedruckt; die Interpunktion dieser beiden Briefe ist aus Verständnisgründen modernisiert.

\section{KARL MARX AN SOPHIE VON HATZFELDT ${ }^{1}$}

12 Sept. 1864.

1, Modena Villas, Maitland Park, Haverstock Hill. London.

Meine liebe Frau Gräfin,

Sie begreifen, wie mich die ganz unvorbereitete Nachricht ${ }^{2}$ von Lassalle's Tod überrascht, bestürzt u. erschüttert ${ }^{3}$ hat. Er war einer der Menschen,

1 Die Originalhandschrift befindet sich im Besitz von Hermann Graf Hatzfeldt auf Schloß Schönstein, Wissen/Sieg. Der Brief besteht aus einem Bogen im Format $224 \times 179,5 \mathrm{~mm}$, ist zu zwei Blättern gefaltet und nur auf der Vorderseite des ersten Blattes von Marx beschrieben. Der Umschlag fehlt. Erstveröffentlichung nach einer 
auf die ich viel hielt. ${ }^{4}$ Es ist mir um so fataler, daß wir in der letzten Zeit nicht mehr in Zusammenhang. ${ }^{5}$ Der Grund war weder allein sein Schweigen ${ }^{6}$ - denn Er begann damit, nicht ich - noch eine mehr als jährige Krankheit, ${ }^{7}$ von der ich erst seit ein paar Tagen frei. Es kommen Gründe hinzu, die ich Ihnen mündlich mittheilen könnte, nicht schriftlich.

Sein Sie überzeugt, daß Niemand tiefern Schmerz über L's Weggerafftsein empfinden ${ }^{8}$ kann. Und vor allem fühle ich für Sie. Ich weiß was Ihnen der Verewigte war, was sein Verlust ${ }^{9}$ für Sie ist. Freuen Sie sich über

Abschrift in F. Lassalle, Nachgelassene Briefe und Schriften, hrsg. von G. Mayer, Stuttgart, Berlin 1921-25 (weiter NBS). Bd 3, S. 408-09. Marx, der die damalige Anschrift der Gräfin in Berlin nicht kannte, ließ den Brief durch W. Liebknecht zustellen, als sie nach der Reise mit Lassalles Leiche in Berlin eintraf (W. Liebknecht an Marx, ca 16.9.1864, Marx-Engels-Nachlaß D 3090, IISG).

2 Die Nachricht von Lassalles Duell (am 28.8.1864 in der Nähe von Genf an der Straße zwischen Carouge und Veyrier) mit J. v. Racowitza wegen H. v. Dönniges und von Lassalles Tod (in Genf am 31.8.) gelangte über G. Bethlen, einen der Sekundanten Lassalles, und über G. Klapka an F. Freiligrath nach London (1. bzw. 2.9.). Freiligrath informierte sogleich Marx, dieser Engels in Manchester. Freiligrath an Marx, 1. und 2.9., in Freiligraths Briefwechsel mit Marx und Engels, bearb. und eingel. von M. Häckel, Berlin 1968, Bd I, S. 176f.; Marx an Engels, 2.9., in K. Marx, F. Engels, Werke, Berlin 1957-68 (weiter MEW), Bd 30, S. 427; vgl. I. Britschgi-Schimmer, Lassalles letzte Tage, Berlin 1925; M. F. Compagnon, ,Le duel et la mort de Ferdinand Lassalle (Genève, août 1864)", in: Revue d'Histoire Suisse, Bd 21 (1941), S. 79-115.

3 In ihren unmittelbaren Reaktionen auf den Duellausgang zeigten sich Freiligrath ,tief ergriffen” (an Marx, 1.9., vgl. aber Marx an seine Frau, 2.9.), Marx „bestürzt” und Engels ,überrascht" (an Marx, 4.9.), MEW, Bd 30, S. 429, 671.

4 Engels an Marx, 4.9.: „Lassalle mag sonst gewesen sein, persönlich, literarisch, wissenschaftlich, wer er war, aber politisch war er sicher einer der bedeutendsten Kerle in Deutschland.” Marx an Engels, 7.9.: „Er [Lassalle] war doch noch immer einer von der vieille souche und der Feind unsrer Feinde." (ebd., S. 432)

5 Dieser Zusammenhang war seit zwei Jahren gelockert. Lassalles Besuch in London von Juli bis Anfang August 1862 hatte Marx deutlich gemacht, daß es zusätzlich zu den politischen Kontroversen und wissenschaftlichen Divergenzen auch noch ein tiefes menschliches Mißverständnis zwischen ihnen gab. Mayer, Einführung in NBS, Bd 3, S. 21f.; H. Oncken, Lassalle. Eine politische Biographie, 4. Aufl., Berlin 1923, S. 272f., 352-73; Sh. Na'aman, Lassalle, Hannover 1970, S. 496-99.

${ }^{6}$ Lassalle hatte nach Marx' bitterem Brief vom 20.8.1862 über eine zwischen ihnen vereinbarte Wechseltransaktion die freundschaftlichen Beziehungen unterbrochen und Marx" einlenkendes Schreiben vom 7.9. nicht mehr beantwortet (MEW, Bd 30, S. 633, 636f.). Die Gräfin war über die Gründe des Zerwürfnisses unterrichtet (s. Anm. 18).

7 Für Marx' Furunkulose s. Nr 4, S. 237.

8 Seine Empfindungen für Lassalle versuchte Marx der Gräfin noch einmal mitzuteilen in dem als Fragment überlieferten Brief vom 16.10.1864 (MEW, Bd 31, S. 419), der eine Antwort (s. Anm. 21) auf den hier als $\mathrm{Nr} 2$ wiedergegebenen Brief darstellt.

9 Zur „Identität des innersten geistigen Wesens”, die Lassalle mit der Gräfin verband, und zum Außergewöhnlichen dieser Verbindung s. Oncken, Lassalle, a.a.O., S. 67f., 77f. 
eins. Er ist jung gestorben, im Triumph, als Achilles. ${ }^{10}$ Ich hoffe, meine liebe Frau Gräfin, daß Sie mit Ihrem hohen u. tapfern Geist diesen Schicksalsschlag ertragen, u. stets überzeugt sind von der vollkommen treuen Ergebenheit

Ihres aufrichtigen Freundes Karl Marx

2. SOPHIE VON HATZFELDT AN KARL MARX ${ }^{11}$

\section{Berlin}

\section{October 1864}

Lieber Marx, Ihr Brief war mir ein wahrer Trost. Ihre Worte gingen mir zum Herzen, weil sie offenbar aus dem Ihrigen kamen. ${ }^{12}$ Ich kann jetzt noch keine langen Briefe schreiben, zu sagen, wie schrecklich mich dieser Schlag getroffen, wie er mich vernichtet hat, wäre ja ohnehin unmöglich. Für solchen Verlust gibt es keine Zeit, er bleibt ewig von gestern für mich. Es ist mir nichts dabei erspart geblieben, auch in den Détails. Ich habe nur noch ein Geschäft in dieser Welt, seine Vertheidigung, sein Andenken so hoch $u$ erhaben zu stellen, daß keine Verläumdung mehr heranzureichen ${ }^{13}$ vermag. Aber auch dafür begegne ich überall Hindernisse in der Ausfüh-

10 „Er starb jung - im Triumphe - als Achilles”, stand als Motto - unter Hinweis auf Marx’ Brief an die Gräfin - über dem Lassalle-Nekrolog in der Probenummer des Social-Demokrat, Organ des Allgemeinen deutschen Arbeiter-Vereins, Red. J. B. v. Hofstetten und J. B. v. Schweitzer, Berlin, 15.12.1864, S. 2. Marx' Würdigung, die die Gräfin bewegte (s. Nr 2-3), wurde im Text des Nekrologs durch mehrfache Paraphrasierung abgewertet (Marx an Engels, 13.3.1865, MEW, Bd 31, S. 99). Anhand der öffentlichen Verwendung seiner in einer privaten Kondolenz gemachten Äußerung erkannte Marx, daß er für die Lassalle-Apotheose benutzt werden sollte, $s$. W. Liebknecht an Marx, 20.12.1864, Marx-Engels-Nachlaß D 3099.

11 Kopie der Originalhandschrift im Marx-Engels-Nachlaß D 2252, Original im IML Moskau.

12 Nach Liebknecht hatte Marx' Kondolenzbrief der Gräfin ,viel Freude” gemacht. Sie war jedoch noch immer erzürnt, weil Lassalle von Marx ,im Stich gelassen” worden sei (an Marx, ca 30.9.1864, Marx-Engels-Nachlaß D 3091). Marx kommentierte, er habe Lassalle keinen „,bessern Dienst” erweisen können, ,als das Maul zu halten und ihn gewähren zu lassen" (an Engels, 4.11., MEW, Bd 31, S. 10).

13 Bei der Verwirklichung ihres Schwurs, Lassalles Tod zu rächen, sein Andenken zu ehren und sein Werk fortzusetzen (s. Anm. 15), ging die Gräfin eigentümliche Wege. W. Rüstow war von einer ,tiefen mentalen Störung" der Gräfin überzeugt. ,Von einem Tag zum andern war bald dieser bald jener ein wahrer Freund und in der nächsten Stunde wieder ein Feind ohne gleichen." (an J. Ph. Becker, 16.5.1865, J. Ph. Becker-Nachlaß D II $900 \mathrm{~b}$, IISG) 
rung (z.B. die zu schreibende Brochure; ${ }^{14}$ das ganze Bild steht so klar vor mir, jedes Wort, das er gesprochen, ist in mein Gehirn eingegraben), aber zu schreiben vermag [2] ich nichts. Die ganze Partei, ${ }^{15}$ die seit seinem Tode reißend zunimmt $u$ in der größten Rachewuth $u$ Begeisterung für ihn ist, den sie als den neuen Erlöser betrachten, verlangt stürmisch von mir eine Parteischrift, eine Apotheose ${ }^{16}$ für ihn. Er soll im Grabe noch fortwirken.

14 Ferdinand Lassalle. Dokumentarische Darstellung seiner letzten Lebenstage. Von Augenzeugen und Freunden, Berlin 1865 (B. Andréas, Ferdinand Lassalle - Allgemeiner Deutscher Arbeiterverein. Bibliographie ihrer Schriften und der Literatur über sie 1840 bis 1975, Bonn 1981 (weiter FL-ADAV), S. 137, B l; zum Schicksal dieser fast gänzlich vernichteten Schrift s. Britschgi-Schimmer, Lassalles letzte Tage, a.a.O., S. 12-28). Die Gräfin ließ die Broschüre hauptsächlich von Liebknecht (s. Anm. 23) redigieren. Vorher hatte sie zwei Lassalle-Freunde, die als Augenzeugen selbst Publikationsabsichten hatten, brüskiert. J. Ph. Becker, der unmittelbar auf die Lassalle-Angriffe der liberalen Presse, z.B. der Neuen Frankfurter Zeitung (s. den Exkurs, S. 237f.), mit seiner Darstellung Die letzten Tage von Ferdinand Lassalle (FL-ADAV, S. 183, C 100) antworten wollte, hinderte sie, weil „Beleidigungen, Verleumdungen, Hinterlist, Komödie u. Betrug gegen Lassalle" zu wenig herausgearbeitet seien (S. v. Hatzfeldt an Becker, 29.10.1864, Becker-Nachlaß D I 933). W. Rüstow, der eigene Aufzeichnungen zur Verfügung gestellt hatte (an Becker, 26.1.1865, ebd., II 899), sah die Hauptfehler der Broschüre in der „Verhimmelung” Lassalles und in dem krampfhaften Bemühen, nachzuweisen, Lassalle habe H. v. Dönniges nicht geliebt; er erhob Einspruch gegen den Druck (an R. Schlingmann, nach dem 18.2., Sammlung ADAV/Hatzfeldt (weiter $\mathrm{ADAV} / \mathrm{H}$ ) 262, IISG). Am 22./23.2. schlossen der Verleger, mit dem die Gräfin sich ebenfalls zerstritten hatte, und diese einen Vergleich über das Nichterscheinen der Broschüre (ebd., 267).

15 Die Gräfin spricht nicht vom „Verein”, sondern von der „ganzen Partei”, die sich zu Lassalle bekenne, um die Sinnlosigkeit von Überlegungen, die auf Marx' Übernahme der Präsidentschaft zielten, anzudeuten. Liebknecht, dem sich andere anschlossen, hatte nämlich Marx unmittelbar nach Lassalles Tod gefragt, ob er nach Deutschland kommen und die "Leitung des Vereins" übernehmen wolle (an Marx, ca 2.9.1864, Marx-EngelsNachlaß D 3089). „Unter gewissen Bedingungen” erklärte sich Marx am 12.9. bereit (Karl Marx. Chronik seines Lebens in Einzeldaten, Moskau 1934, S. 230). Noch am 4.10., als Marx' Interesse sich schon auf die Internationale Arbeiterassoziation (s. Anm. 104) gerichtet hatte, ließ er wissen, daß er es für eine ,gute Parteidemonstration" halte, wenn er gewählt würde, aber öffentlich ablehnen könnte (an C. Klings, 4.10., MEW, Bd 31, S. 417f.). Die Gräfin rechnete es sich als Verdienst an, die Pläne der „Marxianer, die Marx an die Spitze bewegen wollten, womit die Organisation Lassalle's zertrümmert" würde, vereitelt zu haben (an Becker. 29.10.). Trotz ihres Bestrebens, Marx für den Lassalle-Kult zu gewinnen, stand ihre Uberzeugung fest: „Marx, sein [Lassalles] politischer Antipode, intriguirt unablässig, seine Erbschaft anzutreten. Dazu muß er die Organisation auflösen, damit, wie er sagt, ,der Verein in die Partei, d.h. Person Marx, aufgehe. Wo ist die Partei? Wo war sie 15 Jahre lang [. . .]?" (an Becker, 10.3.1865, Becker-Nachlaß D I 938) Zum Parteiverständnis innerhalb des ADAV s. A. Herzig, Der Allgemeine Deutsche Arbeiter-Verein in der deutschen Sozialdemokratie, Berlin 1979, Kap. 2-3.

16 Marx, der den vorliegenden Brief Engels zur Kenntnis schickte, hatte die Absicht der Gräfin sogleich durchschaut und ihr am 16.10. ,sehr freundlich, aber dennoch diplomatisch abwehrend geantwortet" (s. Anm. 21). Er fügte hinzu: „Der moderne Erlöser! Die Person und die Sykophanten, die sie umgeben, sind toll." Den Apotheose-Verdacht übertrugen Engels und Marx auch auf den in der Gründung begriffenen Social-Demo- 
Dies ist auch mein Zweck, meine Anschauung, aber zur Ausführung überall Hindernisse, Rücksichten auf Feinde, Indifferente usw., Verständigkeit, petites morales. ${ }^{17}$ Ich leide noch neben meinem tiefen Kummer noch viel, und derjenige, der immer wußte, konnte, großartig Alles anschaute, er ist nicht mehr da, keiner versteht mich mehr, $u$ ich bin hilflos. Und doch $m u ß$ ich, denn nur dazu hat er mich zurückgelassen. Daß Ihnen das Mißverständniß, ${ }^{18}$ das in der letzten Zeit zwischen Ihnen gewaltet, jetzt sehr traurig, ist mir sehr natürlich.

[3] Er hatte Sie wahrhaft lieb u ließ Ihnen so gern volle Gerechtigkeit widerfahren bei jeder Gelegenheit, wie freudige Anerkennung ein Grundton überhaupt seiner großangelegten Natur war. Und Sie haben gewiß auch Alles Große u Gute, was in ihm lag, gewußt; es strahlt jetzt um so heller, seitdem der Tod die kleinen, vom irdischen Sein unzertrennlichen Flecken von ihm genommen hat. Liebknecht sagt mir, es sei wahrscheinlich, daß Sie bald nach Holland ${ }^{19}$ gingen, würden Sie sich nicht entschließen, alsdann auch nach Berlin ${ }^{20}$ zu kommen? Mir würde es großer Trost sein, Sie sind ja einer seiner ältesten Freunde.

Leben Sie wohl, lieber Marx, u seien Sie meiner herzlichen Freundschaft ${ }^{21}$ versichert

S. v. Hatzfeldt

kraten. Marx an Engels, 4.11., Engels an Marx, 7. und 16.11., MEW, Bd 3I, S. 10, 17, 23, sowie Anm. 10.

17 Über „Schwierigkeiten, nüchterne Ausbesserungen, Verständigkeits- und Moralitätsrücksichten” bei der Abfassung der .,Partei- und Vertheidigungsschrift, eine Apotheose und ein Racheschrei" beklagte sich die Gräfin auch in ihrem Brief an Emma Herwegh vom 5.10. (Ferdinand Lassalle's Briefe an Georg Herwegh. Nebst Briefen der Gräfin Sophie Hatzfeldt an Frau Emma Herwegh, hrsg. von M. Herwegh, Zürich 1896. S. 118f.). Dabei unterschätzte sie die rechtlichen Probleme, wenn sie z.B. Korrespondenzen W. und H. v. Dönniges' mit veröffentlichen wollte (R. Schlingmann an W. Rüstow, 10.2.1865, ADAV/H 263, s. ferner für die Kriterien. nach denen Liebknecht redigierte, Britschgi-Schimmer, Lassalles letzte Tage, S. 16-17).

18 Der Briefwechsel zwischen Lassalle und Marx zur italienischen Frage hatte der Gräfin vorgelegen, daher datierte sie die politische Trennung zwischen beiden auf 1859. Sie war auch darüber informiert, daß Lassalle sich mit Marx wegen einer „Geldangelegenheit, die selbst dem gutmüthigen Lassalle zu stark wurde", völlig entzweit hatte. S. v. Hatzfeldt an Becker, 1.4.1865, Becker-Nachlaß D I 939.

19 Marx wollte mit den drei Töchtern im August 1864 Verwandte in Zaltbommel besuchen, die Reise fand aber nicht statt (Marx an L. Philips, 25.6., an Engels, 25.7. und 31.8., MEW, Bd 30, S. 666, 421, 423).

20 Marx hätte, wie im Frühjahr 1861 , mit einem ihm verfügbaren $\mathrm{Pa} ß$ vorübergehend nach Berlin kommen können, durfte sich aber nicht in Preußen ansiedeln, weil seine Renaturalisation, die Lassalle und die Gräfin betrieben hatten, von der preußischen Regierung wegen fehlender ,royalistischer Gesinnungen” abgelehnt worden war (S. v. Hatzfeldt an Marx, 14.6.1861, Marx-Engels-NachlaB D 2249; Lassalle an Marx, 1.7., NBS, Bd 3, S. 366-70). 
3. SOPHIE VON HATZFELDT AN KARL MARX ${ }^{22}$

Meine Adresse

Berlin 21. Nov.

ist jetzt

1864

Behrenstraße $\mathrm{N}^{0} 11$

Lieber Marx, ich schreibe heute nur zwei Zeilen in rasendster Eile. Ich bin Tag u Nacht beschäftigt mit der Durchsicht der Brochure über Lassalle's Tod. Liebknecht ${ }^{23}$ schreibt sie, u sie ist so weit, daß in drei Tagen der

21 Für das inkonstante Freund-Feind-Denken der Gräfin s. Anm. 13, für Marx' diplomatische Abwehrs. Anm. 16. Bei J. Ph. Becker beklagte sich die Gräfin am 29. 10., daß sie keine Freunde um sich finde. Sie habe ,,verzweiflungsvoll an Marx nach London geschrieben, ihn beschworen zu kommen, er ist krank." Später gab sie Marx' Argumentation, die nicht in seinem Brieffragment vom 16.10. überliefert ist, wieder. Marx hatte demnach geantwortet. die Gräfin könne die Broschüre allein fertigstellen; sie brauche nur jemanden, der aufsetze, was sie sage, ,es müsse aus dem Herzen geschrieben sein, die menschliche Seite, die Hauptsache hierbei, menschlich erklärend." (an Becker, vor dem 11.12., Becker-Nachlaß D I 943) Becker erklärte sich am 11.12. der Gräfin gegenüber mit Marx' Ansicht einverstanden (ADAV/H 6/8). Wahrscheinlich führte Marx' Rat an die Gräfin zur Beauftragung Liebknechts. Wesentlich ist, daß Marx nur noch von der „menschlichen" Seite, nicht aber vom Parteischriftcharakter der Broschüre sprach. Zu der dialektischen Beziehung zwischen Marx und der Gräfin s. H. Hirsch, Sophie von Hatzfeldt, Düsseldorf 1981, Kap. 5-6.

22 Kopie im Marx-Engels-Nachlaß D 2253, Original im IML Moskau.

${ }^{23}$ Liebknecht hatte die Redaktionsarbeit nach L. Buchers und B. Beckers gescheiterten Versuchen und nach Marx' Absage bei aller Sympathie für die Gräfin nur unwillig als Parteipflicht übernommen (B. Becker, Enthüllungen über das tragische Lebensende Ferdinand Lassalles. Auf Grund authentischer Belege, Schleiz 1868, S. III-IV (zur Geschichte dieser Broschüre vgl. FL-ADAV, S. 138f., B 4); Wilhelm Liebknecht, Briefwechsel mit deutschen Sozialdemokraten, Bd 1, 1862-1878, hrsg. und bearb. von G. Eckert, Assen 1973, S. 30, Anm. I, Auszug aus dem Brief an Marx, 5.12.. Marx-EngelsNachlaß D 3097). Anfang Februar $1865 \mathrm{kam}$ es in der Broschürenangelegenheit aus Anlaß von Rüstows Intervention (s. Anm. 14) zwischen der Gräfin und Liebknecht zu Verstimmungen, Mitte Februar zum Bruch (Wilhelm Liebknecht, Briefwechsel mit Karl Marx und Friedrich Engels, hrsg. und bearb. von G. Eckert, Den Haag 1963, S. 41, 43; Briefwechsel mit deutschen Sozialdemokraten, a.a.O., S. 43. Anm. 1, Auszug aus dem Brief an Marx, 16.2., sowie S. 51, Anm. 4). Das politische Zerwürfnis war kurz vorher erfolgt, als Liebknecht von der Bismarck-Faszination der Gräfin (vgl. Botschafter Paul Graf von Hatzfeldt, Nachgelassene Papiere 1838-1901, hrsg. und eingel. von G. Ebel, Boppard/Rh. 1976) und von Lassalles Bismarck-Verhandlungen erfahren hatte. Er berichtete an Marx: „Die Gräfin hat sich (durch Wagner [sic]) dem Bismarck zur Verfügung gestellt, und ihm - in partibus - die ganze ,Partei' Lassalle, Verein und alles verhandelt. Bismarck sollte $d$. allg. direkte Wahlrecht bewilligen, ein paar sozialistische Konzessionen machen, und dafür agitierten wir für die Annexion Schl. Holsteins an Preußen. Der gemeine Plan ist Lassalle's own - sein ,Vermächtnis'." (vor dem 25.1., MarxEngels-Nachlaß D 3102) Für erste Reaktionen von Marx und von Engels - ,objectiv war es eine Schufterei” - vgl. Marx an Engels, 25.1., Engels an Marx, 27.1., MEW, Bd 31, S. 43. 46; ferner G. Mayer, Bismarck und Lassalle. Ihr Briefwechsel und ihre Gespräche, Berlin 1928. 
Druck $^{24}$ beginnt. Es ist eine weit längere u schwerere Arbeit, als ich gedacht, den durch die Briefe fortlaufenden Faden festzuhalten u die vielen Erlebnisse, die in so kurzen Tagen gedrängt sind, klar zu machen. Und wie furchtbar leide ich bei diesen Recapitulirungen! Mein Leben ist zu Ende, die Kugel traf mich eben so tödtlich, nur daß mir die schlimme Todes Art wurde, daß ich mich langsam innerlich verbluten muß. Sie [2] wissen, wie wir oft im Scherz früher von meiner Rachsucht sprachen, Sie können sich denken oder vielmehr Sie können sich nicht denken, mit welcher verzehrenden intensität mich dieses Rachebedürfni $\beta^{25}$ jetzt plagt. Ich bin ihm Rache schuldig, ich $m u ß$ es können, sonst bin ich ein miserables Geschöpf $u$ verachte mich selbst.

In die Brochure soll, wie man von allen Seiten von mir fordert, die Photographie ${ }^{26}$ Lassalle's auf dem Todtenbett vorn kommen, die Arbeiter verlangen dieses Andenken. Weiter hinten in der Brochure an den betreffenden Stellen, wo sie auftreten, die beiden Mörder. ${ }^{27}$ Finden Sie das gut, daß die drei Bilder hinein kommen? Freilich wenn Sie mir darüber etwas sagen wollten, müßten Sie es [3] gleich ${ }^{28}$ thun. Sollen Unterschriften außer den Namen unter die Bilder? und welche? Vielleicht unter Lassalle's Bild Ex ossibus ultor, ${ }^{29}$ er hatte nämlich in den letzten Monaten unbewußt Todes Ahnungen, die sich in der sonderbarsten Weise überall zeigen; in

24 Am 25.11. schloß die Gräfin mit R. Schlingmann einen Vertrag, wonach 1800 Exemplare der Broschüre in erster Auflage gedruckt werden sollten. Verlagsvertrag ADAV/H 264.

25 Das Rachebedürfnis, das bei ihr und den Anhängern Lassalles herrschte, wollte die Gräfin durch die Verfolgung H. v. Dönniges' und J. v. Racowitzas als „,Mörder” Lassalles befriedigen (G. Mayer, „Gräfin Sophie von Hatzfeldt, Bismarck und das Duell Lassalle-Racowitza", in: Historische Zeitschrift, Bd 134 (1926), S. 47-56). Für Marx' geschicktes Eingehen auf die Rachegedanken der Gräfin s. Nr 4, für Lassalles Rachemotiv Anm. 30.

${ }^{26}$ Einige Stunden nach seinem Tod wurde Lassalle in seinem Sterbebett auf Veranlassung der Gräfin photographiert, der Genfer Bildhauer F.-G. Dufaux nahm ihm die Totenmaske ab (Britschgi-Schimmer, Lassalles letzte Tage, S. 292; Compagnon, ,Le duel et la mort", a.a.O., S. 100). Für die Broschüre ließ die Gräfin 1600 Exemplare des Leichenbildes herstellen (vgl. den in Anm. 14 erwähnten Vergleich). Ein Exemplar der Lassalle-Broschüre mit dem genannten Bild befindet sich im Besitz von B. Andréas, Versoix.

27 Die Gräfin hatte, seit ihrem Scheidungsprozeß mit detektivischen Methoden vertraut, kurz nach dem Duell das Signalement J. v. Racowitzas verbreitet und sich auch die Photographien von ihm und von $H$. v. Dönniges zu verschaffen gewußt (S. v. Hatzfeldt an Becker, 11.9. und 17.10., Becker-Nachlaß D I 927, 930, ferner ihre Bemerkungen zum Verlagsvertrag ADAV/H 266). Außerdem ließ sie Racowitza durch Louis Weber verfolgen (Marx an S. Meyer, 27.8.1867, MEW, Bd 31, S. 558).

28 Siehe Anm. 46.

29 Worte der Dido in Vergils Aeneis, Buch IV, Vers 625. 
allen seinen letzten Reden ${ }^{30}$ spricht er von seinem Untergang $u$ führt diese Worte an! Oder die Worte aus einem Ihrer Briefe, die mir so gefielen:

$$
\begin{gathered}
\text { „Er starb jung - im Triumpf } \\
\text { wie Achilles". }
\end{gathered}
$$

Kurz, wenn eine Unterschrift sein soll oder kann, geben Sie mir eine an.

Was soll unter die beiden anderen Bilder?

Unter das Bild der infamen Dirne ${ }^{31}$ vielleicht die Worte Lassalle's in seinem letzten Brief an sie, den sie aber nicht mehr erhalten hat, wo er schon halb aufgeklärt über sie, und welche lauten: [4] „Mein Blut (oder mein Schicksal, ich habe den Brief nicht zur Hand) komme über Dich, u mein Fluch verfolge Dich bis in's Grab." 32 Oder was sonst?

Unter das Bild des Mörders kann, scheint mir, nichts anderes kommen können als sein Name und: „tödtete Ferdinand Lassalle 28ten August 1864 zu Genf." ${ }^{33}$ Finden Sie das auch? Ich verbinde mit dem Erscheinen der Portraits der Mörder in der Brochure Rachezwecke, ihre Schande an ihnen überall persönlich zu kennzeichnen, Haß u Rache bei den Anhängern L's noch mehr zu entflammen u vorzüglich mir die Auffindung und Verhaftung des Rakowitza zu erleichtern. ${ }^{34}$

Ach, hätten Sie doch jetzt hier sein können und dem Freunde den

30 Am Schluß der Ronsdorfer Rede hatte Lassalle seine Todesahnung ausgesprochen und das „Exoriare aliquis nostris ex ossibus ultor” übertragen mit „Möge, wenn ich beseitigt werde, irgend ein Rächer und Nachfolger aus meinen Gebeinen auferstehen!" Die Versammelten waren laut Lassalles effektvollem Vermerk zum Schwur aufgefordert worden. In der Broschüre sollte das Ende der Ronsdorfer Rede hinter Lassalles Photo (s. Anm. 26) als Motto erscheinen. Das Vergil-Zitat hatte Lassalle auch anläßlich seiner Geburtstagsfeier im Berliner ADAV am 11.4.1864 vorgetragen. F. Lassalle, Gesammelte Reden und Schriften, hrsg. und eingel. von E. Bernstein, Berlin 1919-20, Bd 4, S. 228f; Ferdinand Lassalle. Dokumentarische Darstellung, a.a.O., Motto und S. 75. Nach Lassalles Tod hallte die Parole „Ex ossibus ultor” von allen Seiten wider; G. Herwegh schrieb sogar ein Gedicht unter dieser Überschrift, das die Gräfin namentlich zeichnete und das als Inschrift für Lassalles Sarg vorgesehen war (Nordstern. Organ für das deutsche Volk, 24.9.1864, S. 1).

31 Für die Beurteilung H. v. Dönniges' vgl, ihre beiden Schriften (H. v. Racowitza, Meine Beziehungen zu Ferdinand Lassalle, Breslau 1879; dies., Von Anderen und mir, Berlin 1909), in denen sie bemüht war, der Gräfin die Schuld am verhängnisvollen Duell zuzuschieben. Von ,verworfener Dirne” war in Lassalles Brief an W. v. Dönniges, 26.8.1864, in dem auch die Duellforderung stand, die Rede (Britschgi-Schimmer, Lassalles letzte Tage, S. 27lf., ebd. auch die Kritik an H. v. Dönniges' Memoiren, S. 297-310).

${ }^{32}$ Lassalle an H. v. Dönniges, 20.8.: „Aber wenn Du mich zerbrichst durch diesen bübischen Verrath, den ich nicht überwinde, so möge mein Loos auf Dich zurückfallen und mein Fluch Dich bis zum Grab verfolgen." Ferdinand Lassalle. Dokumentarische Darstellung, S. 149.

33 Diese Unterschrift hätte gleich zwei Unrichtigkeiten enthalten (s. Anm. 2).

34 Vorstehender Satz bildet im Brief der Gräfin eine Anmerkung am linken Briefrand. Wegen des Rachethemas s. Anm. 27. 
letzten Liebesdienst erweisen können, sein Andenken zu schützen u zu ehren!

Nun zu dem eigentlichen Zweck meines heutigen Briefes. Dieser infame Schuft, der Carl Blind, hat sich erfrecht, im deutschen Blatt in London oder als Flugblatt dort gedruckt, es steht auch im Francfurter Journal, einen schändlichen Angriff auf Lassalle zu machen; es ist betitelt Republikanischer Protest ${ }^{35}$ es wurde mir heute geschickt, ich kann es Ihnen aber nicht mitschicken, da hier darauf [5] eine Erklärung ${ }^{36}$ im Namen des ganzen Vereines gemacht werden soll. Sie werden es aber leicht in London auftreiben, auch nach America $^{37}$ hat er es verbreitet. Lassalle lebend hätte darüber gelacht, darüber brauchte $e r$ sich nicht mehr zu vertheidigen, aber dem Todten darf es nicht ungestraft angethan werden. Freilich soll ja dem todten Löwen der Eselstritt nicht fehlen dürfen. ${ }^{38}$ Konnte Lassalle dafür, $\mathrm{da} ß$ es noch so total blödsinnige Leute gibt, daß sie die Unterscheidung $z w i s c h e n$ dem Staat $u$ diesem Staat $^{39}$ nicht zu verstehen vermögen. Aber dieses Subject ist wohl nicht so total blödsinnig, sondern einfach ein Schurke, der noch nicht vor langer Zeit die namenlose Nichtswürdigkeit hatte, am Tage, nachdem Garibaldi ${ }^{40}$ öffentlich erklärt, daß er nichts so ${ }^{35}$ Der ,Republikanische Protest” war in der Neuen Frankfurter Zeitung vom 29.9., S. 3. erschienen; für seine Verbreitung und Bedeutung s. den Exkurs.

${ }^{36}$ Eine ADAV-Erklärung scheint nicht erfolgt zu sein. Außer Marx' Blind-Erklärung (s. Anm. 105) erschien noch F. Reusche, „Der blinde badische Diplomat und seine neueste Reaktions-Entdeckung", in: Nordstern, 17.12., S. 2-3.

37 Unter der Überschrift ,Ein republikanischer Protest, London 17. Sept. 1864" erschien eine weitere Fassung von Blinds Lassalle-Attacke in der Westlichen Post (St Louis), Ende Oktober oder Anfang November. Ein exakter Nachweis konnte wegen der bruchstückhaften Überlieferung dieser deutschamerikanischen Zeitung, zu der Blind über K. Schurz Verbindung hatte (s. K. Blind, „Karl Schurz”, in: Die Gartenlaube, 1906, S. 489), nicht geführt werden. Seine Existenz ist bezeugt von J. Weydemeyer, der ihn Engels zusandte, und von Marx, der ihn für seine Blind-Erklärung benutzte (Weydemeyer an Engels, Oktober 1864, Weydemeyer-Nachlaß 254, IISG; Marx an Engels, 25.11., MEW, Bd 31, S. 31, sowie Anm. 105).

${ }_{38}$ Marx nimmt diese Anspielung auf Aesops Fabel „.Der vom Alter entkräftete Löwe” in seiner Blind-Erklärung wieder auf (MEW, Bd. 16, S. 24).

${ }^{39}$ Lassalle wollte sich "dieses” Staats, d.h. des vorhandenen preußischen, monarchischen Staats, ,als eines unentbehrlichen Mittels” bedienen, um „den Staat”, d.h. den „demokratischen Nationalstaat" mit seinen ,großen sozialen Zukunftsaufgaben” herbeiführen zu helfen (Oncken, Lassalle, S. 365). Vgl. auch Lassalles Äußerungen über den Staatszweck des Arbeiterstandes am Anfang und - über die mögliche Rolle des preußischen Königtums, ,wahrhaft große nationale und volksmäßige Ziele zu verfolgen” - am Ende seiner Agitation (F. Lassalle, Das Arbeiterprogramm; ders., Der HochverrathsProceß, in Gesammelte Reden und Schriften, Bd 2, S. 194-200; Bd 4, S. 159). Einen Ansatzpunkt zu dieser Entwicklung sah Lassalle im Sommer 1864 in der SchleswigHolstein-Frage (s. Anm. 42).

${ }^{40}$ Garibaldi hatte bei seiner Großkundgebung im Londoner Kristall-Palast am 16.4 . 1864 (für Garibaldi in London s. Anm. 76) von Preußen und Österreich gesprochen, die die Landung in Neapel gern verhindert hätten und die nun ,poor little Denmark” 
sehr bedauere, als daß er seinen Degen [6] nicht Dänemark weihen könne, mit einer deutschen Deputation ${ }^{41}$ im Gepränge zu ihm zu gehen, seine ekelhaften Lobhudeleien anzubringen. Diese Creaturen sind es, die den deutschen Namen im Ausland so verachtet machen. Man weiß ja nicht, daß es nicht wirklich so misérable bei uns aussieht, $u$ daß wahrlich Garibaldi heute in Deutschland sicher weder Deputationen noch Ovationen ${ }^{42}$ zu gewärtigen hätte.

Ich überliefere also hiermit diesen Blind für seine letzte Schandthat Ihren Händen, lieber Marx, ergreifen Sie Ihre spitzeste, giftigste Feder u

niederdrücken wollten. "It is sad that two colossi are soon trying to put down a little country that has no assistance from any one, and has only his native strength to resist the invaders." (The Times, 18.4.. S. 9) Blind hatte vor der Rede auf Garibaldi im Sinne einer prodeutschen Schleswig-Holstein-Lösung (s. Anm. 42) einzuwirken versucht und wurde von Freiligrath, der zur deutschen Garibaldi-Delegation (s. Anm. 78) gehörte, sogleich kritisiert. Garibaldi scheine die bei Blind ,genommene Lection schon vergessen zu haben. Wie konnte er sich nur so ausdrücken?! Österreich u. Preußen - preisgegeben! Natürlich! Aber ,little Denmark?!' Und von der eigen tlichen, brennenden Frage, von den Herzogthümern u. deren Analogie mit Venetien, kein Wort! Ich verstehe dies nicht, vollends, wie gesagt, nicht nach Ihrer Mittheilung am Freitag Abend, die inzwischen, mehr oder weniger ausführlich, auch schon die Mehrzahl der deutschen u. englischen Blätter gebracht hat." An Blind, 18.4., Blind Papers, Vol. 2, f. 310, British Library, London; für Blinds Reaktion auf diese Kritik s. Anm. 41.

41 Die deutsche Deputation war am 19.4. mit Garibaldi zusammengetroffen. Blind begrüßte den General als ,the representative of revolution and national independence”. Als Demokraten und Liberale würden sie nichts gemein haben mit den Herrschern von Österreich und Preußen, ,who were tyrants at home, unjust to other nations, and whose acts in the Duchies were impressed with the stamps of treachery". Vom Nationalitätenproblem her betrachtet, sei der Fall Venetien derselbe wie der Fall Schleswig-Holstein. Blind erinnerte daran, Garibaldi ,had declared to him in repeated interviews that on the day when the German nation did unfurl her banner, he (Garibaldi) would be on the side of the oppressed people of the Duchies”. Garibaldi antwortete u.a.: ,As to the Duchies, I neither desire to see Denmark oppressing them, nor can I wish that a little kingdom should be invaded on its own soil by two great despots". [K. Blind?] "Garibaldi and the Germans", in: The Morning Advertiser (London), 20.4., S. 6; s. ferner Anm. 78.

42 Garibaldi hatte seine Londoner Stellungnahme (s. Anm. 40) zwei Tage vor der Erstürmung der Düppeler Schanzen abgegeben. Die preußisch-österreichischen Truppen hatten gesiegt und am 31.10. war der Friede mit Dänemark, das die Herzogtümer abtreten mußte, geschlossen worden (s. [E. Bauer,] „Schleswig-holsteinischer Krieg”, in: Staats- und Gesellschafts-Lexikon, hrsg. von H. Wagener, Berlin 1865, Bd 18, S. 311-15). Eine garibaldinische Lösung des Schleswig-Holstein-Konflikts war durch diese Ereignisse unmöglich geworden. Lassalle hatte noch vor seiner Reise in die Schweiz die Gräfin über seinen geplanten „Coup” in der Schleswig-Holstein-Frage unterrichtet. Er wollte auf einer großen Arbeiterversammlung in Hamburg am 25.9. die Resolution durchsetzen, „daß Bismarck verpflichtet sei, die Großherzogthümer zu annexieren gegen den Willen Österreichs und der übrigen Staaten". S. v. Hatzfeldt Lassalle zitierend an Becker, 1.4.1865; Liebknechts Version im Brief an Marx, vor dem 25.1., s. Anm. 23; zum Hamburger „Coup” ferner E. Bernstein, Ferdinand Lassalle. Eine Würdigung des Lehrers und Kämpfers, Berlin 1919, S. 288; Na'aman, Lassalle, a.a.O.. S. 750-54. 
vertheidigen Sie Lassalle u vernichten Sie dieses Subject! ${ }^{43}$

Nun leben Sie wohl, lieber Marx, es ist vier Uhr Nachts, u ich falle um vor Müdigkeit. Aus den paar Zeilen ist ein langer gekritzelter u, fürchte ich, sogar unverständlicher Brief geworden. Sie antworten mir gewiß trotz Ihrer Beschäftigungen gleich auf meine Fragen. Mit den herzlichsten Grüßen

Ihre S. v. H.

Liebknecht grüßt Sie herzlichst, er hat in diesen Tagen keine Minute Zeit zum Schreiben. Ich will den C. Blind auch bei Garibaldi u Mazzini44 gehörig besorgen.

\section{KARL MARX AN SOPHIE VON HATZFELDT ${ }^{45}$}

\section{Meine liebe Frau Gräfin!}

24 Nov. 1864.

1, Modena Villas, Maitland Park, Haverstock Hill, London.

Ich habe Ihnen so eben per Telegraph ${ }^{46}$ (damit ja d. Antwort sich nicht verspäte) meine Ansicht mitgetheilt, daß unter keinen Umständen die beiden Photogramms v. R. et Co. ${ }^{47}$ gleichzeitig mit dem v. Lassalle in d.

43 Die Gräfin konnte auf Marx' Bereitschaft zählen, gegen Blind öffentlich aufzutreten, da sie über Blinds Rolle in der Vogt-Affäre und Marx' Feindschaft ihm gegenüber unterrichtet war (s. Anm. 54).

44 Die Gräfin und Lassalle hatten Garibaldi im November 1861 auf Caprera besucht; Lassalle traf im Juli 1862 in London mit Mazzini zusammen. Diese Kontakte wurden belastet durch Lassalles Ansinnen, die zukünftigen Aktionen der beiden italienischen Nationalisten für seine nationalrevolutionären Ziele auszunutzen. Außerdem waren diese Treffen zu sporadisch gewesen, um die festeren Beziehungen von Blind, der den Emigrantenführern die vermeintliche Persigny-Rolle Lassalles vorzeichnete, zu lockern (s. Anm. 62-63).

45 Die Originalhandschrift befindet sich im Besitz von Hermann Graf Hatzfeldt. Der Brief besteht aus 3 Bogen im Format $218 \times 178 \mathrm{~mm}$, ist zu 6 Blättern gefaltet und auf 12 Seiten von Marx beschrieben. Der Umschlag fehlt.

46 Das Telegramm ist bislang nicht bekannt geworden. B. Becker behauptete später, daß Marx „Telegramme und Briefe" an die Gräfin schickte, um sie gegen den ADAV aufzubringen. Marx stellte daraufhin richtig, daß es sich nur um dieses eine Telegramm handelte, in dem er die Frage nach der ,Zugabe gewisser Porträts zur beabsichtigten Broschüre” mit „Nein” beantwortet habe. „Der ,Präsident der Menschheit”" (1865), MEW, Bd 16, S. 93, vgl. auch Marx an Engels, 24.11.1864, MEW, Bd 31, S. 30, wo Marx seine Ablehnung unterstreicht.

47 Gemeint sind J. v. Racowitza und H. v. Dönniges. 
Broschüre erscheinen dürfen. Es wäre dieß einerseits eine Verunehrung des Dahingeschiedenen u. gäbe andrerseits der Schrift ein buntes Aussehn, das ganz u. gar zu vermeiden ist. Im übrigen wissen Sie, daß auch mein Motto ist: „contra hostem vindicatio aeterna sit”, 48 aber um die Rache mit Erfolg auszuüben ist es rathsam das Publicum nicht in die rachsüchtige ,Absicht"49 einzuweihn.

„Ex ossibus ultor” scheint mir die beste Unterschrift unter L's Porträt. Es war ebenfalls das letzte Wort, das ich vor ungefähr einem halben Jahr meinem unvergeßlichen Freunde W. Wolff ${ }^{50}$ in das Grab nachwarf.

Was den Ex-studiosus ${ }^{51}$ Blind betrifft, diesen Burschen, der vor 1848 als Colporteur Heinzen'schen Unsinns ${ }^{52} \mathrm{u}$. als homme entretenu der jüdischen Banquiersfrau Cohen ${ }^{53}$ (die er später in London heirathete) sich bekannt

48 Wohl dem Rechtssatz „Adversus hostem aeterna auctoritas [esto]" des altrömıschen Zwölftafelgesetzes (III 7) nachgebildet; ,vindicatio” ist von Marx im Sinne von „Rache” gebraucht.

49 Siehe Nr 3 und Anm. 25.

50 Wilhelm Wolff war am 9.5. in Manchester gestorben. Marx hielt beim Begräbnis am 13.5. eine ,kleine Grabrede”. Karl Marx. Chronik, a.a.O., S. 228; MEW, Bd 30, S. 659; ferner W. Schmidt, Wilhelm Wolff. Kampfgefährte und Freund von Marx und Engels, Berlin 1979, S. 310 f.

51 Bei seiner Agitation im Sommer 1847 wurde Blind in der Presse als „Student” vorgestellt, Deutsche-Brüsseler-Zeitung 1. Januar 1847 - 27. Februar 1848. Faksimile mit Einführung und Anmerkungen von B. Andréas, J. Grandjonc und H. Pelger, Brüssel 1981, 9.9.1847, S. 2. Blind hatte u.a. mit G. A. Schlöffel und J. Miquel in Heidelberg studiert und agitiert. J. H. Schoeps, „Im Kampf um die deutsche Republik. Karl Blind und die Revolution in Baden 1848/49", in: Revolution und Demokratie in Geschichte und Literatur. Zum 60. Geburtstag von Walter Grab, hrsg. von J. H. Schoeps und I. Geiss, Duisburg 1979, S. 259-61; für Schlöffel s. G. Rasch, ,Ein Streiter der Revolution”, in: Der Deutsche Eidgenosse, hrsg. von K. Blind u.a. (London und Hamburg, Reprint Glashütten/Ts 1973), 15.7.1865, S. 82-88, sowie Miquel an Marx, Sommer 1850, in K. Marx, F. Engels, Gesamtausgabe (MEGA), Dritte Abteilung, Bd 3, Berlin 1981, S. 592. Für Marx und Blind s. auch G. Mayer, „Letters of Karl Marx to Karl Blind”, in: International Review for Social History, Bd 4 (1939), S. 153-59.

52 Der „Student Blind und Frau Cohen" wurden wegen Verbreitung der Flugschrift Deutscher Hunger und deutsche Fürsten verhaftet. Der Aufruf wurde zeitgenössisch fälschlicherweise als kommunistisch betrachtet. Solcher „Unsinn”, stellte die DeutscheBrüsseler-Zeitung, 12.9.1847, S. 3, richtig, ,ist kraß. Der Aufruf wurde uns von Karl Heinzen zugeschickt, und bekanntlich hat Heinzen mehrmals den Kommunismus angegriffen". (Das Flugblatt war abgedruckt worden ebd., 20.6., S. 1. Wegen eines weiteren Abdrucks in der Deutschen Londoner Zeitung und wegen Heinzens Verfasserschaft vgl. die Einführung in die Deutsche-Brüsseler-Zeitung, S. 68-69, Anm. 141. Schoeps, „Im Kampf um die deutsche Republik”, S. 261, hat die Flugschrift noch, J. Peiser, „Gustav Struve als politischer Schriftsteller", Phil. Diss. Frankfurt 1973, S. 45, folgend, Blind zugeschrieben.) Für Blinds Bezıehung zu Heinzen s. Anm. 87.

53 Friederike Ettlinger aus Karlsruhe, verwitwete Cohen (F. Lautenschläger, „Karl Blind”, in: Neue Deutsche Biographie, Bd 2, S. 304f.), ,eine von jenen deutschen Frauen [...], welche mehr Entschiedenheit besitzen, als 50 Maulhelden und Festessen-Liberale 
machte, so bitte ich Sie sich zu erinnern,${ }^{54} \mathrm{da} B$ ich in meiner Schrift: ,, Herr Vogt" (Siehe p. 58-69 u. Beilagen 9, und 11) ${ }^{55}$ diesen studiosus [2] Blind nicht nur als ,,infamen Lügner" charakterisire, sondern daß ich durch gerichtliche Aktenstücke nachweise, daß dieser Elende ,falsche Zeugniße” schmiedete, um mich zu verdächtigen u. seinen Kopf aus einer Schlinge zu ziehn, die dieses Subject im Interesse bloßer Wichtigthuerei ${ }^{56}$ (dieß ist in der That der einzige Zweck dieses Strolchs in allen seinen öffentlichen Schritten od. vielmehr Kriechungen) sich selbst gelegt hatte.

Es gelang Blind damals die Katastrophe seines politischen Treibens abzuwehren, - durch bloßes Stillschweigen. ${ }^{57}$ Er wurde dabei unterstützt

zusammen” (Deutsche-Brüsseler-Zeitung, a.a.O.). F. Cohen, eine „lebhafte Jüdin”, war Feuerbach-Anhängerin (Marx an Engels, 13.10.1851, MEW, Bd 27. S. 357f.) und Goethe-Kennerin (O. Bielefeld, ,Karl Blind in London”, in: März, Jg. 1 (1907), Bd 3, S. 354). Auch später, als ihr zweiter Mann, K. Blind, Mitglied des Bundes der Kommunisten geworden war, nahm sie an der politischen Tätigkeit Anteil (C. Schramm an Marx. 9.9.1850, in MEGA, a.a.O., S. 632). Im Londoner Exil verkehrten die Familien Blind und Marx eine Zeit lang freundschaftlich miteinander (s. Anm. 96). Für den ersten Mann Friederikes, einen ,,banker of independent means, elderly and widowed", sowie für die Ungleichheit der Ehepartner ,,both in age and temperament" s. die biographische Skizze in The Poetical Works of Mathilde Blind, ed. by A. Symons with a memoir by R. Garnett, London 1900.

54 Marx hatte Lassalle und auch der Gräfin, die zusammen 80 RT zur Drucklegung von Herr Vogt zugeschossen hatten (Lassalle an Marx, 30.10.1860, NBS, Bd 3, S. 341), je ein Exemplar übersandt. Lassalle dankte für den „,herrlichen Genuß" mit Brief vom 19.1.1861. ,Wir haben wie toll gelacht, als ich es vorlas, die Gräfin und ich. Sie dankt Dir aufs herzlichste für das Buch. Seit lange hatte sie sich nicht so gut amüsiert, als bei dieser Lektüre." (ebd., S. 347)

${ }_{55}$ K. Marx, Herr Vogt, London 1860, MEW, Bd 14, S. 473-89, 672, 673. Marx beschreibt in der von ihm angegebenen Stelle die „Augsburger Campagne”. Blind hatte Marx am 9.5.1859 über Vogts „,bonapartistische Subsidien” und „landesverräterische Umtriebe” informiert. Diese Nachricht gelangte nicht durch Marx, sondern durch E. Biscamp und Liebknecht in die Presse, woraufhin Vogt gegen die Augsburger Allgemeine Zeitung wegen Liebknechts Londoner Korrespondenz Klage erhob. Blind, zur Beweisführung aufgefordert, erklärte, ,gar kein Anteil" an der Affäre zu haben und suchte seine Nichtbeteiligung mit Falschaussagen anderer (s. Anm. 75) zu belegen. Bei Beilage 9 handelt es sich um einen Artikel Blinds, mit dessen Hilfe Marx ihn als Verfasser des anonymen Flugblatts gegen Vogt identifizierte, und Beilage 11 stellt einen Auszug aus Marx' Zirkular gegen Blind vom 4.2.1860 dar, in dem Marx von einer ,wilfully false declaration in the Augsburg Gazette" und von ihrem Verursacher Blind als einem „deliberate liar” sprach.

56 Siehe Anm. 105.

57 Blind, der in freundschaftlicher Verbindung zu Freiligrath stand, hatte diesem nach Marx' Brief an Freiligrath vom 23.11.1859 (Freiligraths Briefwechsel, a.a.O., S. 121-23) vorgeschlagen, auf Schreiben von Marx und Liebknecht „einstweilen nicht einzugehen”, um nicht ,neues Material zu Insinuationen" zu liefern (an Freiligrath, nach dem 23.11., Blind Papers, Vol. 4, ff. 92-93). 
einerseits dadurch, daß Freiligrath (dieß entre nous!) leider fortfuhr öffentlich als sein Freund ${ }^{58}$ zu figuriren, u. daß Lassalle vernachlässigte, wozu ich ihn dringend aufgefordert hatte, d'une manière ou d'une autre das deutsche Publicum mit der Nase auf meine An-den-Pranger=Stellung B's zu stoßen. ${ }^{59}$ Mir schien das nothwendig, weil die deutsche lib. Presse instinktiv mit einem Lumpen wie B. ging, also meinen Angriff todtzuschweigen suchte. Lassalle hielt den Blind für zu unbedeutend. Er lernte erst später ${ }^{60}$ kennen, daß man dann u. wann den ,Kampf mit einer Wanze"61 nicht abwehren kann.

Als Lassalle hier in London war, suchte er auf seinen persönlichen Besuchen bei Louis Blanc ${ }^{62}$ u. Mazzini ${ }^{63}$ sie [3] über Blind aufzuklären,

58 Freiligrath trat nicht nur öffentlich weiterhin als Freund Blinds auf (s. Anm. 78), sondern stellte „die Freundschaft zu Blind über die zu Marx" (Häckel, Einleitung in Freiligraths Briefwechsel, Bd I, S. XCI; Marx an Lassalle, 7.9.1860, NBS, Bd 3, S. 317). So teilte er Blind mit, daß Marx ihm „,den Krieg erklärt” habe, daß dieser eine „Broschüre” (nämlich den Herrn Vogt) „loslassen" werde und daß man - nach Marx' Besuch bei Lassalle - in Köln .,etwas stutzig über die Übersiedlung des,Père Marx' nach Berlin” sei (an Blind, 2. und 6.2.1860, 12.4.1861, Blind Papers, Vol. 2, ff. 13 If., 133f., 186f.). Vgl. Mayer, „Letters”, a.a.O., S. 155f.; ferner für Freiligrath und Blind noch G. W. Spink, Ferdinand Freiligraths Verbannungsjahre in London, Berlin 1932 (Reprint Nendeln 1967), bes. S. 62f.

59 Als Marx seine Replik auf eine Erklärung Blinds in der Vogt-Affäre (s. Anm. 55) an Lassalle mit der Bitte sandte, dieselbe in der Berliner Volkszeitung unterzubringen, sicherte Lassalle seine Bereitschaft zu, riet aber grundsätzlich von der Veröffentlichung der Replik ab (Marx, ,Erklärung”, 15.11.1859, MEW, Bd 14, S. 692f.; an Lassalle, 15.11.; Lassalle an Marx, 20.11., NBS, Bd 3, S. 236f.). Für Lassalles Haltung in der Vogt-Affäre insgesamt vgl. Oncken, Lassalle, S. 182-84; Mayer, Einführung in NBS, Bd 3, S. 14-18. Lassalle hatte schon 1849 ein distanziertes Verhältnis zu Blind und sprach sich offen gegen Blinds Artikel „Österreichische und preußische Parteien in Baden” in der von Marx redigierten Neuen Rheinischen Zeitung. Politisch-ökonomische Revue (s. Nachdruck mit Einl. von K. Bittel, Berlin 1955, S. 63-69) aus (an Marx, 24.10.1849 und 16.4.1850, NBS, Bd 3, S. 17, 24).

60 Siehe Anm. 103.

61 H. Heine, Atta Troll. Ein Sommernachtstraum (1847), Kap. XI, in Werke und Briefe, Berlin 1961, S. 376: „Ja, das Schrecklichste auf Erden / Ist der Kampf mit Ungeziefer, I Dem Gestank als Waffe dient - / Das Duell mit einer Wanze!"

62 Über die persönliche Beziehung zwischen Lassalle und Louis Blanc ist wenig bekannt (NBS, Bd 5, S. 312). „Eines Tages [wohl im Jahre 1863] wandte sich Lassalle brieflich an ihn [Blanc], um ein Schreiben von ihm zu erhalten, das dem sog. ,revolutionären Agitator' in der deutschen Arbeiterwelt als Geleitschein dienen sollte. Louis Blanc bat mich um meine Meinung, was zu thun sei. Die allgemeine Auffassung ging damals dahin: Lassalle sei ein republikanischer Führer von äußersten Grundsätzen, der eine gewaltige gesellschaftliche Umwälzung anstrebe. Ich meinerseits hielt ihn von Anfang an für einen ehrgeizigen Catilinarier, der im geheimen Einverständniß mit der Regierungsgewalt die freisinnigen Bestrebungen des Bürgerthums zu Falle zu bringen suche, um an ihre Stelle ein als Groß-Almosenier auftretendes, despotisches Königthum zu setzen, das sich in der Weise des zweiten napoleonischen Kaiserreiches mit demokratischen Redensarten und Scheineinrichtungen verbräme. Dieser Ủberzeugung gab ich Louis Blanc gegenüber 
aber vergeblich. Dieser Mensch ist ganz das, was Mazzini, Ledru ${ }^{64}$ etc bei d. andren Deutschen ${ }^{65}$ von Namen umsonst gesucht hatten, ein kriechender Knecht u. Sykophant. Durch sein Verhältniß zu diesen Leuten macht er die Engländer glauben, daß er Deutschld repräsentirt, u. durch seine Windbeuteleien hier imponirt er den Deutschen. Dabei besteht die ganze Thätigkeit dieses Burschen darin, in Verbindung mit 3 od. 4 Süddeutschen, ${ }^{66}$ von, für u. über , Karl Blind ${ }^{\prime 67}$ zu schreiben u. bei allen möglichen Gelegenheiten sein ,unmaßgebliches Urtheil” als selbsterkorner Repräsentant des ,deutschen Republikanismus" ${ }^{68}$ [einer bekannt-

Ausdruck. [. . .] Lassalle wollte eine Persigny-Rolle spielen.” K. Blind, ,Louis Blanc”, in: Die Gegenwart, Bd 23 (1883), S. 4. Dieser Nekrolog gibt auch Auskünfte über die enge Beziehung zwischen Blanc und Blind, vgl. außerdem die diversen Briefe Blancs an Blind in den Blind Papers.

${ }^{63}$ Für die enge Beziehung zwischen Mazzini und Blind s. den umfangreichen Briefwechsel in den Blind Papers, Vol. 1, veröffentlicht als Lettere inedite di Giuseppe Mazzini (il più grande esule) all'esule Carlo Blind, Neapel [1927], und K. Blind, ,Josef Mazzini", in: Die Gegenwart, Bd I (1872), S. 165-66, 181-82, 214-17; ferner Marx an Engels, 25.2. und 4.3.1865, MEW, Bd 31, S. 86, 90, und F. Della Peruta, „I democratici italiani, i democratici tedeschi e l'unità d'Italia (1859-1861)", in Democrazia e socialismo nel Risorgimento, 2. Aufl., Rom 1977, bes. S. 210-13.

${ }^{64}$ Für die enge Beziehung zwischen Blind und Ledru-Rollin vgl. den Briefwechsel in den Blind Papers. Dieser wurde von A. R. Calman, Ledru-Rollin après 1848 et les proscrits français en Angleterre, Paris 1921, nicht benutzt; Blind wird nur unter Hinweis auf seine „Personal Recollections about Ledru-Rollin”, in: Fraser’s Magazine, Neue Serie, Bd 11 (1875), S. 243-53, zitiert; s. ferner ders., ,Erinnerungen an Ledru-Rollin”, in: Die Gartenlaube, 1875, S. 148-51, 160-64; „,Ledru-Rollin. Aus persönlichen Erinnerungen”, in: Die Gegenwart, Bd 13 (1878), S. 161-63.

${ }^{65}$ Siehe Anm. 84-85.

${ }^{66}$ Es handelt sich vor allem um Eduard Bronner und Karl Heinrich Schaible (s. Anm. 74-75).

${ }^{67}$ Für diese Formel, die bei Marx und Engels geläufig wurde, s. den von Engels englisch verfaßten und von Marx überarbeiteten Text im Marx-Engels-Nachlaß H 68, unter der Überschrift „Über Karl Blind” in deutscher Übersetzung veröffentlicht in MEW, Bd 17, S. 267.

68 Blind hatte sich 1849 vor Gericht als Sozial-Republikaner bezeichnet (Verhandlungen der Freiburger Assisen gegen G. Struve und K. Blind, 20-30. März 1849, Extra-Beilage zur Mannheimer Abendzeitung, Nr 1-18, S. 1-72, hier S. 57). 1865, als er den Eidgenossen plante, wollte er damit ein „Kampfblatt für die verschiedenen Fraktionen der republ. $\mathbf{u}$. sozialist. Partei bilden" und bekannte sich in Erinnerung an 1849 als „, Republikaner, das heißt als Social-Demokrat", ,wenn auch an polit. Erfahrungen etwas reicher geworden" (K. Blind an NN, o.D. [1865], Blind Papers, Vol. 4, ff. 114-15). An den „Sozial-Demokraten” Blind von 1849, der „ausschließlich Nationalrepublikaner” geworden sei und sich „öffentlich im Gegensatz zur deutschen demokratischen Partei entschieden für die Annexion von Elsa $B$ und Lothringen" ausgesprochen habe, erinnert 1876 Blinds ehemaliger Freund Amand Goegg ([A. Goegg,] Nachträgliche authentische Aufschlüsse über die Badische Revolution von 1849 [...] Nebst einem Nachtrag, Zürich 1876). Ebenfalls 1876 spricht Blinds ehemaliger Verbündeter G. Rasch (s. Anm. 72) von dem „Sozialrepublikaner” der 48er Revolution, der nicht mehr von der „,deutschen demokratischen und föderalen Republik” spreche, sondern sich als ,guter Deutscher" zum 
lich nur im Mond existirenden Partei, da der deutsche Bourgeois constitutionell monarchisch, der Feudale aristokr. monarchisch, u. der Arbeiter überhaupt nicht für bloße Staatsformen, sondern für die Staatsform als Ausdruck ökonomischer socialer Verhältnisse sich interessirt u. unter allen Umständen nie den Exstudiosus Blind als Vertreter anerkannt hat] Engländern, Deutschen u. Amerikanern aufzudrängen. Er ist ein wahrer Meister darin einem seiner 6 Trabanten ${ }^{69}$ Briefe zu dictiren, worin sie den etc „Karl Blind” zu dem oder jenem auffordern, oder ihm zu dem u. jenem gratuliren, u. dann - durch Versehn od. otherwise - kommen d. Antwortschreiben desselben K.B. in die Presse.

Als einer der ,young men”, die im Dienste F. Heckers ${ }^{70}[4]$ thätig waren, lernte Blind diese unter den Süddeutschen keineswegs seltene Kunst der Selbstbegeifferei u. der Schöpfung einer falschen Public Opinion über an $u$. für sich unbedeutende Persönlichkeiten. In London glaubte er weit genug avancirt zu sein oder lang genug gedient zu haben in der „Demokratie", um die im Dienst Andrer erworbenen Künste für sich selbst anzuwenden.

Was seine Bildung betrifft, so ist sie, wie ich Lassalle bei seinem Hiersein sagte, die eines badischen Wirthes, der Rotteck's Weltgeschichte, Welker's Staatslexicon u. den republikanischen Kalender des Herrn Stru$v^{71}$ gelesen hat.

„Nationalliberalen” gewandelt habe (,Deutsche Flüchtlinge in London”, in: Volksstaat, 30.7., S. 1-2).

69 Marx denkt wohl an den Verein „Deutsche Freiheit und Einheit”, der etwa 1860 in London gegründet wurde und zu dem außer Blind, Bronner (s. Anm. 74) und Schaible (s. Anm. 75) auch noch ein Ravenstein als Schriftführer und ein Wolffsohn als Kassierer (über die beiden letzten ließ sich nichts ermitteln) gehörten. G. Rasch, „Karl Blind”, in G. Struve und G. Rasch, Zwölf Streiter der Revolution, Berlin 1867, S. 139, und die editorische Notiz in Der Deutsche Eidgenosse, 15.3.1865.

${ }^{70}$ Für Blinds Reise nach Straßburg vor der Februarrevolution 1848 im Auftrage F. Heckers und für seine Beteiligung an dessen Aprilaufstand vgl. Schoeps, ,Im Kampf um die deutsche Republik", S. 261-65.

71 Karl von Rottecks Allgemeine Weltgeschichte für alle Stände, 4 Bde, 1835, ein Auszug aus seiner Allgemeinen Geschichte, 9 Bde, 1812-27, war von nachhaltigem aufklärerischen Einfluß auf das „Geschichtsbild des deutschen Bürgertums im 19. Jahrhundert”; das von ihm und Karl Welcker herausgegebene Staatslexikon. Encyclopädie der Staatswissenschaften, 15 Bde, 1834-43, galt als „Lehrbuch des Frühliberalismus” (W. Kosch, Biographisches Staatshandbuch, Bern 1963, Bd 2, S. 1051). Beim „Kalender” handelt es sich um das Republikanische Regierungs-Blatt, hrsg. von G. v. Struve, „,im Namen der provisorischen Regierung Deutschlands", 22.9.1848. Es erschien nur diese Nummer. Der darin enthaltene „Aufruf an das deutsche Volk” mit der Parole „Deutsche Republik! Wohlstand, Bildung, Freiheit für Alle!” war gezeichnet „Der Schriftführer: Karl Blind. Im Namen d. provisor. Regierung Deutschlands: Gustav Struve. Hauptquartier Lörrach, am ersten Tage der deutschen Republik, am einundzwanzigsten September 1848" (s. Rasch, ,Karl Blind”, a.a.O., S. 125f.). Marx’ Bemerkung über die Bildung eines „,badi- 
In Dtschld wird Herr B. in seinen Manövern namentlich unterstützt v. Herrn Gustav Rasch ${ }^{72}$ zu Berlin.

Bernhard Becker, ${ }^{73}$ als ehemaliger subeditor d. Londoner Hermann weiß am besten, wie Blind die über ihn z.B. im Hermann erschienenen Lobhudeleien selbst höchsteigenhändig geschrieben hat. Dieß thut er meistens u. im Durchschnitt. Zur Abwechslung läßt er wohl einmal einen gewissen Dr Bronner ${ }^{74}$ in Bradford od. seinen Schaible ${ }^{75}$ (Sieh „Herr

schen Wirts" enthält auch eine Anspielung auf Blinds Vater Johann Adam, der zunächst Wachsfabrikant, dann Gastwirt in Mannheim war (s. auch Marx an Engels, 25.11.1864). 72 Marx kannte Rasch seit dem Frühjahr 1849 (s. Rasch an Marx, 9.4.1849, in MEGA, a.a.O., S. 343); im Juni 1849 trafen Rasch, Blind und Marx in Paris zusammen (G. Rasch, Aus meiner Festungszeit. Ein Beitrag zur Geschichte der preußischen Reaktion, Pest u.a. 1868, S. 28). Im Herbst 1849 ging Rasch zusammen mit Blind ins englische Exil, kehrte aber dann nach Berlin zurück und wurde in Haft genommen, nachdem er sich vorher für Marx und das Londoner Flüchtlingskomitee eingesetzt hatte, dabei aber auf Marx' Ablehnung gestoßen war (E. Dronke an Engels, 21.2. und ca 7.5.1850, in MEGA, a.a.O., S. 486, 540). Vierzehn Jahre später traf Rasch wieder mit Blind in London zusammen und verfaßte als Ergebnis dieses Wiedersehens die Skizze „Karl Blind” für die Biographiensammlung Zwölf Streiter der Revolution, a.a.O. Später wandte sich Rasch der Sozialdemokratie zu (s. Anm. 68 und Engels an Rasch, Ende November 1876, MEW, Bd 34, S. 229, sowie die Polemik, die sich zwischen Rasch und Schaible entspann, ebd., S. 585f., Anm. 334). Zu Raschs journalistischer Tätigkeit in der Schleswig-Holstein-Frage s. Anm. 84.

${ }^{73}$ Marx bezieht sich auf eine Episode im Juni 1863. Unmittelbar nach Erscheinen von Blinds „Freundeswort” (s. Anm. 103) waren E. Dronke und W. Eichhoff in Manchester zusammengetroffen, um Lassalle gegen Blind zu Hilfe zu kommen. Dronke wollte für Lassalle eine „komplette Biographie dieses Menschen zur gelegentlichen Benutzung à la Julian Schmidt" vorbereiten, und Eichhoff suchte von B. Becker eine ,schriftliche Mitteilung" darüber zu erhalten, ob ,,alle die Sch-Blindschen Artikelchen [. . . zum Teil aus Karl Blinds eigener Feder herrührten", wie B. Becker aus seiner Redaktionstätigkeit am Hermann. Deutsches Wochenblatt aus London bekannt sein mußte. „Mir wurde damals in London erzählt", führte Eichhoff nämlich aus, ,,daß Sie zu einer Zeit, wo Juch [s. Anm. 78] in Deutschland gewesen, die Karl Blindschen Noten nicht zum Druck gegeben hätten, worüber sich Karl Blind in einem an die Redaktion des ,Hermann' gerichteten und in Ihre Hände gelangten Briefe noch dazu extra beschwert habe. Wie steht es hiermit?" (B. Becker an Lassalle, 14.6.1863, NBS, Bd 5, S. 186; Becker teilt Lassalle einen Auszug aus Eichhoffs Brief mit). Becker, der den "Schwindler” Blind „sehr genau" kannte, begann daraufhin, ihn im Berliner Volksfreund anzugreifen, und fragte gleichzeitig bei Lassalle an, ob ihm Dronkes Hilfe genehm sei (ebd., S. 187). Lassalle hielt eine literarische Unterstützung durch Dronke, hinter dem nach Beckers Meinung auch Marx stand, für ,sehr wünschenswert" (B. Becker, Geschichte der Arbeiter-Agitation Ferdinand Lassalle's. Nach authentischen Aktenstücken, mit einer Einl. zum Nachdruck von T. Offermann, Berlin, Bonn 1978, S. 63). Wie die Dronke-Initiative endete, ist unbekannt; Marx zeigte sich jedenfalls enttäuscht darüber, daß Becker nicht unter Verwendung des Herrn Vogt gegen Blind aufgetreten war (s. unten, S. 235).

74 Der Arzt Dr Eduard Bronner lebte seit November 1851 im englischen Exil, seit Juli 1852 in Bradford (Badische Biographien, hrsg. von A. Krieger und R. Obser, Bd 4, Heidelberg 1935, S. 57-59). Seine bislang nicht eindeutig identifizierte Korrespondenz 
Vogt”) über London ihre Namen unter seine Schreibebriefe setzen. Die ganze Agitation dieses Menschen ist nichts als ein Gewebe von Lüge, Selbsttäuschung, Windbeutelei. In diesem Fach steht er jedoch als unerreichter Meister da. Das /5/ Climax seines Geschicks zeigte er bei Garibaldi's Besuch in London. ${ }^{76}$ Erst verbreitet er in den engl. Blättern das Gerücht, daß er ein intimer Freund Garibaldi's sei. Er hatte mit seiner gewöhnlichen Zudringlichkeit von London aus „Adressen” 77 an G. erlassen $u$. Sie wissen, daß G. in seiner Herzenseinfalt jedem Menschen freundlich antwortet. Dann stürzt er auf die Isle of Wight (bevor G. seinen

mit Blind befindet sich in den Blind Papers. Für Bronners politisches und publizistisches Zusammenwirken mit Blind vgl. seine Beteiligung am Verein „Deutsche Freiheit und Einheit" (Anm. 69), seine Mitwirkung bei der Garibaldi-Deputation (Anm. 78) und seine Rolle in der Episode um den Stuttgarter Beobachter (Anm. 105).

${ }^{75}$ K. H. Schaible, Siebenunddreißig Jahre aus dem Leben eines Exilierten. Ein flüchtiges Lebensbild. Zum Andenken an deutsche und englische Freunde, Stuttgart, London 1895. Schaible hatte in der Vogt-Affäre Blind zu entlasten gesucht, indem er sich als Verfasser des gegen Vogt gerichteten Flugblatts „Zur Warnung" ausgab (Marx, Herr Vogt, a.a.O., S. 488.; an Engels, 15.2.1860, MEW, Bd 30, S. 41, s. auch Anm.55). Für den Streit zwischen Schaible, der wie Blind nach 1871 Bismarck-Anhänger geworden war, und Rasch (s. Anm. 72) im Anschluß an dessen Artikel „,Deutsche Flüchtlinge in London", a.a.O., vgl. Engels an Rasch, Ende November 1876, MEW, Bd 34, S. 229-31.

${ }^{76}$ Garibaldi, der sich vom 11. bis 22.4.1864 in London beim Herzog von Sutherland, Staffordhouse, aufgehalten hatte, um die Unterstützung der Engländer für einen Kriegszug gegen Österreich und für den Einmarsch in Venetien zu gewinnen, erlebte einen enthusiastischen Empfang - ,which has never been equalled at any former period" (The Morning Advertiser, 23.4., S. 5) - durch die verschiedenen Klassen der Bevölkerung. Gladstone und die englische Regierung sahen sich dadurch gezwungen, unter dem Vorwand von Garibaldis angegriffener Gesundheit dessen Aufenthalt in England vorzeitig zu beenden (für kritische Kommentare s. Marx an L. Philips, 29.3., an Engels, 19.4., sowie Engels an Marx, 29.4., MEW, Bd 30, S. 650, 390f., 392). Die emotionale Bewegung in der Bevölkerung suchten die verschiedenen Gruppen und Organisationen auszunutzen. So war Marx vom Londoner kommunistischen Arbeiterbildungsverein beauftragt worden, eine Adresse an Garibaldi zu verfassen und eine Arbeiterdeputation anzuführen, was er allerdings ablehnte (an Engels, 19.4.). Die Londoner Gewerkschaften bildeten am 13.4. in einer Versammlung im Whittington Club, Strand, unter Leitung von G. Odgers ein „Working Men's Garibaldi Reception Committee”. Am selben Abend wurde noch in Anwesenheit einer Pariser Arbeiterdeputation ein Spezialkomitee gegründet, ,who will have power to convene a public meeting of the working classes for the official reception of the address and the French deputation" (The Times, 14.4., S. 14), eine Station, die hin zur Gründung der IAA am 28.9. führte (Marx an Engels, 4.11., a.a.O., S. 13; H. Hirsch, ,Aufstieg und Niedergang der Ersten Internationale”, in Denker und Kämpfer. Gesammelte Beiträge zur Geschichte der Arbeiterbewegung, Frankfurt/M. 1955, S. 139; weiter s. Anm. 104).

77 Noch bevor eine deutsche Deputation (s. Anm. 78) gebildet worden war, hatte Blind wegen eines Empfangs an Garibaldi geschrieben. Dieser antwortete am 5.4. von der Insel Wight aus und erklärte sich einverstanden (an Blind, Isle Wight, Brooke House, 5.4., Blind Papers, Vol. 2, f. 308; ,Garibaldi and the Germans in England", in: The Morning Advertiser, 8.4., S. 6, in welchem Artikel Blind die Zuschrift Garibaldis verwertete). 
festlichen Einzug in London hielt) u. ,verabredet" mit ihm an welchem Tag G. die deutsche Deputation ${ }^{78}$ empfangen werde [Blind hatte es nämlich so arrangirt, daß er sich von ein paar Deutschen wählen ließ als Führer einer Deputation, wobei Kinkel u. leider auch mein Freund Freiligrath] u. schickt zugleich geheimnißvolle Winke in ein paar Londoner Blätter, daß G. was ,closeted"79 with ,his friend" (Blind) u. natürlich sehr wichtige Staatssachen mit ihm verabredete. Aber B's Meistercoup kömmt erst. Als Bedienter der europ. „Demokratenkönige" 80 (in dieser Form faßt er Mazzini, Ledru Rollin, Louis Blanc, u. in der That, compared with Studiosus Blind sind selbst sie ,große Männer"81) weiß der badische Pfifficus es einzurichten, daß er als Gesellschafter u. Führer den beim Herzog von Sutherland wohnenden Garibaldi abholt, um ihn, im Staatswagen des Herzogs, zu Ledru Rollin und Louis Blanc zu geleiten. Bei der Gelegenheit richtet er es so ein, daß der Wagen am Hause des Herrn Blind stehn bleibt u. Garibaldi ,pays his compliments to [6] Madame Karl Blind”. Alles das erscheint sogar in der Times $^{82}$ durch geschicktes management $u$. in einem

${ }^{78}$ Das deutsche Garibaldi-Komitee wurde am 6.4. in Seydt's Assembly Rooms, Finsburysquare, gebildet. Ihm gehörten u.a. an: Blind als Sprecher und seine Mitarbeiter Schaible und Ravenstein, E. Juch, Verleger des Hermann, der Blind vorgeschlagen hatte, der Verleger N. Trübner, bei dem Blinds Flugblätter erschienen, ferner Freiligrath und G. Kinkel; insgesamt umfaßte es 23 Personen. Kinkel hielt die Lobrede auf Blind, ,who had laboured for so many years past so energetically and faithfully in the cause of international freedom, whilst none, at the same time, had shown more zeal in his own country's cause" (The Morning Advertiser, 7.4., S. 5). E. Bronner berief in Bradford eine Versammlung ein, die Blind mit der Überbringung ihrer Adresse beauftragte (ebd, 14.4., S. 3). Zum deutschen Garibaldi-Komitee und zu Blinds Auftrag s. ferner The Observer, 10.4., S. 3, zum Zusammentreffen der Deputation mit Garibaldi s. Anm. 41.

79 Blind traf am 9.4. mit Garibaldi in Brooke House, Insel Wight, zusammen, beide drückten ,,sentiments of fraternity” aus. „,Karl Blind then remained closeted with Garibaldi for some time. At the wish of the German Committee, the Address will be presented at the General's residence in London." The Morning Advertiser, 11.4., S. 5; für das Verhältnis Garibaldi-Blind s. ferner K. Blind, „Lücken in Garibaldi's Denkwürdigkeiten", in: Das Magazin für die Litteratur des In- und Auslandes. Wochenschrift der Weltlitteratur, Jg. 57 (1888), S. 221-26, $245-49$ (hier auch die Beschreibung der Episode, die Marx den Bouret-Streich nennt, s. Anm. 83), sowie S. 261-64 und ders., "Eine Erinnerung an Garibaldi", in: Vossische Zeitung, 24.7.1903, Morgenausgabe.

${ }^{80}$ Auf Blind zurückgehende Wortschöpfung, die von Marx verschiedentlich persifliert wurde (an Engels, 10.3.1865, MEW, Bd 31, S. 95). Andererseits benutzte Marx „Arbeiterkönige" auch in seriösem Sinn (an Weydemeyer, 29.11.1864, ebd., S. 428).

81 Anspielung auf K. Marx und F. Engels, Die großen Männer des Exils (1852), MEW, Bd 8, S. 233-335, in welchem Pamphlet Mazzini, Ledru-Rollin und Blanc des öfteren erwähnt wurden.

82 ,A great many visitors continued to call upon Garibaldi up to 10 o'clock, shortly after which the General left in the Duke of Sutherland's carriage, accompanied by Mr. Karl Blind, to pay visits. The General drove first to the residence of $\mathrm{Mr}$. Karl Blind, in Townshend-road, St. John's-wood, where he paid his respects to Mrs. Karl Blind, and the 
Augenblick wo G. der Gott Londons. Sie erinnern sich vielleicht wie „Rameau”, d. Neffe, in der wundervollen Schrift Diderot's, in Bewunderung ausbricht über das Genie Bouret's, des Generalpächters, der das Geheimniß wußte seinen kleinen Hund sich abspänstig u. dem Minister zuwendig zu machen! Diese Garibaldi=Komödie war Blind's Bouretstreich! ${ }^{183}$ Ich glaube, seit der Zeit fing er an, sich selbst bona fide für einen großen Mann zu halten! Hat sich was mit Schleswig Holstein! Wissen Sie denn nicht, daß Blind seit Jahren seine Hauptwichtigkeit aus seiner „Vertretung” Schleswig Holstein's gegen Dänemark zieht, daß ,er” die deutschen Tyrannen zum Krieg gegen Dänemark ,gezwungen”, u. daß Freund Rasch ihm daher s. Schrift über den ,verwaisten" Bruderstamm ${ }^{84}$ gewidmet hat! Wissen Sie nicht, daß Prof. Virchow ${ }^{85}$ in der pr. Kammer

members of her family. Thence the General proceeded to call on M. Ledru Rollin, in St. John's-wood-park, and subsequently upon M. Louis Blanc in Melina-place, in the same neighbourhood, with both of whom he had short interviews. On leaving Louis Blanc Garibaldi returned direct to Stafford-house." The Times, 19.4., S. 11, ferner The Morning Advertiser, 20.4., S. 6. und The Observer, 24.4., S. 3.

83 E.-M. Bouret (gest. 1777), Sohn eines Lakaien, als Generalsteuerpächter zu Reichtum gekommen, Mäzen der Anti-Philosophes, wußte sich die Zuneigung seines Protektors, des Generalfinanzkontrolleurs und Siegelbewahrers $M$. d'Arnouville dadurch zu vergrößern, daß er in kurzer Zeit sein Hündchen - das dem Siegelbewahrer gefiel, aber vor ihm Angst hatte - durch Maske. Verkleidung und Dressur dazu brachte, seinen Herrn zu fliehen und d'Arnouville nachzulaufen. Dies gelang Bouret, indem er selbst sein Hündchen schlecht behandelte, in der Maske d'Arnouvilles aber sein Vertrauen gewann. D. Diderot, Oeuvres, hrsg. von A. Billy, Paris 1951, S. 431; ders., Rameaus Neffe und Moralische Erzählungen, übertragen von R. Rütten [Das erzählerische Gesamtwerk, Bd 4], Berlin 1967, S. 73, 273.

${ }^{84} \mathrm{G}$. Rasch, Vom verlassenen Bruderstamm. Das dänische Regiment in SchleswigHolstein, Bde 1-2, Berlin 1862, Bd 3, 1863. Der von uns eingesehene Bd 1 ist nicht Blind gewidmet. Bd 2 aber A. v. Baudissin, Bd 3 R. v. Bennigsen. Es ist aber auch möglich, daß Marx hier auf Raschs Blind-Skizze anspielt, in der Blinds Londoner PropagandaTätigkeit für die Rechte Deutschlands, Italiens und Polens und besonders SchleswigHolsteins, „des von Deutschland vierzehn Jahre lang verlassenen Bruderstamms", außerordentlich gelobt wird (Rasch, „Karl Blind”, a.a.O., S. 137). Raschs SchleswigHolstein-Titel hatten den Charakter von Sensationsschriften, wurden von dänischer Seite angegriffen und erhielten weite Verbreitung; er schrieb außerdem Vom verrathenen Bruderstamm. Der Krieg in Schleswig-Holstein im Jahre 1864, 2 Bde, Leipzig 1864.

85 Bei der Diskussion um den Fonds für das literarische Büreau hatte R. Virchow die Unfähigkeit der Regierung hervorgehoben und angeführt, ,daß gegenüber der Dänischen Agitation ein einziger Privatmann, ein Deutscher Flüchtling, Karl Blind, mehr ausgerichtet hat in der Englischen Presse, als alle Deutschen Regierungen zusammen" (Stenographische Berichte über die Verhandlungen der [. . . ] beiden Häuser des Landtages, Haus der Abgeordneten, 13. Sitzung, 9. Dez. 1863, Berlin 1864, Bd 1, S. 347). Blind wie Marx erfuhren darüber aus der Volks-Zeitung, Berlin, 10.12.1863. Siehe ferner Blind, „Einiges über Virchow”, in: Deutsche Revue, Jg. 27 (1902), S. 304-09, hier S. 305. 
(wahrscheinlich durch Herrn Rasch gespornt) Blind's wichtigen Einfluß in d. Schles. Holst. Sache als Beweis anführte, was ein einzelner Mann durch Energie für ein ganzes Volk thun könne! Der schamlose Mensch hatte die deutschen Esel glauben gemacht, daß er die Englische Ansicht über Schles. Holst. [7] modificirt habe! Seine Wischiwaschiflugblätter ${ }^{86}$ über Schles. Holst. ist ungefähr (u. das heißt viel) das Dümmste, was in dieser line erschienen ist. [Nebenbei! Der beste Freund u. Associirte des Herrn ExStudiosus ist der Lümmel Karl Heinzen ${ }^{87}$ in America, der seit $20 \mathrm{~J}$. ein Geschäft daraus macht mich zu verläumden in einer Weise, wovon selbst Lassalle kein Beispiel erlebt hat. Ich habe es nie der Mühe werth gehalten, diesem Subject ein Wort zu antworten, war aber doch erstaunt, daß der Nordstern $^{88}$ (lange Zeit auch ein Hauptorgan des Studiosus Blind) ftwhd

${ }^{86}$ Eine Zusammenstellung bietet die in Anm. 69 genannte editorische Notiz. Ein Teil dieser Flugblätter ist im Stadtarchiv Mannheim und in der Sammlung Schaible der Universitätsbibliothek Heidelberg erhalten. Marx hatte sich im Laufe des Jahres 1864 wieder intensiv mit den Problemen von Schleswig-Holstein befaßt (Karl Marx. Chronik. S. 229), und Engels war sogar in die Herzogtümer gereist, um sich an Ort und Stelle zu überzeugen. Anders als Lassalle entschieden sie sich gegen eine preußische Annexion und für eine gerechte Lösung durch die Teilung Schleswigs (vgl. den Überblick von G. Beier, „Marx, Engels, Lassalle und der deutsch-dänische Konflikt 1848 und 1864”, in: Grenzfriedenshefte, 1962, S. 194-202, 232-44).

${ }^{87}$ Für Blind, der jahrelang Korrespondent von K. Heinzens Pionier, und für Freiligrath, der Heinzen lebenslang freundschaftlich verbunden war, s. C. Wittke, Against the Current. The Life of Karl Heinzen (1809-1880), Chicago 1945, S. 99, 118. Heinzen und Blind kannten sich seit 1847 (s. Anm. 52). Für Blinds und Heinzens Aufenthalt in Straßburg s. O. Wiltberger, Die deutschen Flüchtlinge in Straßburg 1830-1849, Berlin, Leipzig 1910 , S. 121 passim. 1849 trafen Marx, Blind und Heinzen in London zusammen. Marx und Heinzen hatten sich Ende Oktober 1842, kurz nachdem Marx die Redaktion der Rheinischen Zeitung übernommen hatte, kennengelernt. Der Bruch erfolgte, als Heinzen unter Einfluß A. Ruges im September 1845 einen antikommunistischen Artikel veröffentlichte (H. Pelger, ,Karl Marx und die rheinpreußische Weinkrise”, in: Archiv für Sozialgeschichte, Bd 13 (1973), S. 329-32). Die Beziehungen und Polemiken zwischen Marx und Heinzen, die mehr als dreißig Jahre dauerten, können hier nur skizziert werden. Marx und Engels attackierten Heinzen 1847 in der Deutschen-Brüsseler-Zeitung (s. die diversen Angaben in der Einführung) und im Pamphlet Die großen Männer des Exils, a.a.O., S. 282-87; weiter forderte Marx J. Weydemeyer und A. Cluß auf, Heinzen in Amerika publizistisch anzugreifen. Heinzen schrieb u.a. Die Helden des teutschen Kommunismus. Dem Herrn Karl Marx gewidmet, Bern 1848, und verfolgte Marx in seinen deutschamerikanischen Blättern Janus und Pionier sowie in der deutschen liberalen Presse.

88 Organ radikaler Hamburger Demokraten, seit Januar 1860 erschienen und seit Ende 1860 von K. v. Bruhn redigiert. Blind, der v. Bruhn seit dem Hecker-Putsch kannte, war so lange der wichtigste Mitarbeiter, bis der Nordstern im Zuge der Kongreßbewegung sich an Lassalle anschloß. Auch scheint Blind. ähnlich wie beim Hermann, an der Finanzierung vermittels des Revolutionsfonds beteiligt gewesen zu sein. Als Blind im Juni 1863 sein „Freundeswort” (s. Anm. 103) an die Redaktion nach Hamburg schickte, fand es keine Aufnahme mehr. Ab August 1863 unterstützte Lassalle den Nordstern 
die Sudeleien des Heinzen, die den Principien d. Arbeiterpartei auf das Feindlichste gegenüberstehn, abdruckt, u. überhaupt Propaganda für Herrn Heinzen macht.]

Aber Garibaldi, Schleswig Holstein, America, Lassalle, alles ist für den Ex-Studiosus nur ein Vorwand sich selbst wichtig zu machen! Der Bescheidene schrieb vor einiger Zeit höchsteigenhändig an d. „Glasgow Sentinel", 89 daß ganz Europa (wörtlich) sich noch nicht in zwei feindliche Lager mit Bezug auf „Karl Blind” gespalten habe, sondern daß ein tiers parti auch noch in dieser Rücksicht existire! Im „Observer" 90 bezeichnet er sich selbst als ,the illustrious chief of the German Republicans".

Zur weiteren Charakteristik dieses in der That, wenn er richtig aufgefaßt wird, hochkomischen Charakters, noch einige kleine Züge.

finanziell (v. Bruhn an Lassalle, 22.6., NBS, Bd 5, S. 192f., 265-68: Marx an Engels, 15.8., MEW, Bd 30, S. 369). Heinzens Pionier-Artikel waren häufig im Nordstern nachgedruckt worden. Auch legte v. Bruhn Wert darauf, daß Heinzen mittels Broschüren über Lassalles Agitation informiert wurde. Anders als Blind, dessen „Hochachtung” für die Fortschrittler er kritisierte, war Heinzen mit Lassalle „fast ganz”- d.h. nicht mit dessen Klassenkampf- und Arbeiterbewegungs-Vorstellungen - einverstanden. v. Bruhn an Lassalle, 10.5.1864, NBS, Bd 5, S. 310f.; zu v. Bruhn und Heinzen s. T. Offermann, Arbeiterbewegung und liberales Bürgertum in Deutschland 1850-1863. Bonn 1979, bes. S. 297,460 .

${ }^{89}$ Marx hatte auf diesen Artikel, der in North British Daily Mail (Glasgow) erschienen war, bereits in seinem Brief an Engels hingewiesen (an Engels, 9.4.1863, MEW, Bd 30, S. 341-42). Blind war im März 1863 während seiner Polen-Agitation auch nach Glasgow gefahren und wertete sich (der Artikel war gezeichnet „Communicated”) in der liberalen Zeitung des ihm befreundeten Verlegers John McAdam folgendermaßen: „German by birth and German by exile, M. Karl Blind's efforts have not come so prominently and so persistently before Europe, as to have gained for him universal admiration from the liberating party, or universal execration from the oppressing party. He has hitherto stood in that middle way, where he has the honour of being both beloved and hated; but in these two contending ranks which have rendered to him their tribute after its kind the whole of Europe is not ranged, M. Karl Blind having the satisfaction of knowing that in Europe there is a third section of his friends who are simply indifferent. He therefore comes before the Scottish public with perhaps less prejudice against him than has been the case with most of the distinguished exiles who have preceded him.” „M. Karl Blind”, in: North British Daily Mail, 30.3., S. 4; im Original keine Hervorhebungen, vgl. aber den obenerwähnten Brief von Marx, in dessen Abschrift auch „that in Europe" fehlt. Der hieraus hervorgegangene Artikel „Karl Blind in Schottland”, in: Hermann und Nordstern, beide vom 25.4, veranlaßten Dronke und Eichhoff zu ihrer Initiative gegen Blind (s. Anm. 73).

90 The Óbserver, 10., 17. und 24.4.1864, jeweils S. 3, hatte über Blinds Rolle in der Garibaldi-Deputation berichtet. Dabei wird Blind in London als „speaker and representative of Germans here" bezeichnet. Bei Erwähnung der von E. Bronner veranlaßten Bradford-Deputation heißt es: „The long-established personal friendship between the General and Karl Blind, and the consonancy of their political sympathies, naturally causes the latter to be chosen to express to the former the admiration and good wishes of the German Liberals residing in this country." 
Nach seinem Erfolg mit Garibaldi Mr. Karl Blind joined, wie im Athenäum zu lesen stand, the Shakespeare Committee!91 [8] Er war offenbar nun auch zum ,literarischen” Repräsentanten Dtschlands avancirt!

Während d. badischen Crawalls (1849) schickte Struve, wie er selbst in einer Schrift über die „,bad. Revolution” erzählt, den ,jungen Mann”, wie er den Blind nennt, als Untersecretär des zum Secretär ernannten Schütz aus Mainz nach Paris, um den Ex Studiosus Blind zu retten vor Collision mit Brentano. ${ }^{92}$ Die Regierung, an die Blind als Untersecretär des Secretär Schütz adressirt war, - die provisorische Regierung existirte nicht mehr als Secretär u. Untersecretär in Paris ankamen. ${ }^{93}$ Dennoch hat Blind in ge-

y1 „Herr Carl Blind and M. Amédée Pichot have joined the National Shakespeare Committee." (The Athenaeum. Journal of English and Foreign Literature, Science. and the Fine Arts (London), 2.4.1864, S. 511) Bei den Vorbereitungen zum "Shakespeare Festival" anläßlich von Shakespeares 300 . Geburtstag - gefeiert vom 23.4. bis 3.5.1863war es zu Streitigkeiten im Komitee gekommen, die Personalergänzungen notwendig machten. Freiligrath war bereits vorher zum Komitee-Mitglied ernannt worden (ebd.. 20.2., S. 268) und hatte wohl für Blinds Aufnahme gewirkt. Garibaldis London-Besuch begann am 11.4., Blinds Auftritt dabei fand also nach - und nicht, wie Marx vermutet. vor - der Berufung ins Shakespeare-Committee statt (Marx an Engels, 19.4., a.a.O., S. $390)$.

${ }^{22}$ G. Struve, Geschichte der drei Volkserhebungen in Baden, Bern 1849, S. 193. Blinds Handexemplar befindet sich im IISG, Sign. D 1120/173, und weist Streichungen oder Bemerkungen an den Stellen auf, die Blind betreffen; so bei folgender Bemerkung Struves: „Nichts destoweniger blieb der Widerwille Brentano's gegen Blind bestehen. Unter diesen Umständen nahm Carl Blind nicht ungern die Stelle eines Gesandtschaftssekretärs in Paris an". Blind korrigierte Struve, indem er das ,nicht” vor ,ungern” strich.

93 Die pfälzisch-badische Mission von F. Schütz (zur Person s. die Einführung in die Deutsche-Brüsseler-Zeitung, S. 26 und Anm. 50, 148) und Blind, die die Unterstützung der französischen Republik für die beiden deutschen provisorischen Regierungen erzielen sollte, ist von Blind bei jeder Gelegenheit in immer neuen Variationen dargestellt worden. Eine knappe Übersicht der Ereignisse gibt Schoeps, ,Im Kampf um die deutsche Republik”, S. 274-76. Blind war kurz nach Schütz, zusammen mit Ruge (K. Blind, .Zur Erinnerung an Arnold Ruge”, in: Die Gegenwart, Bd 19 (1881), S. 97-100; ders., „Ruges Geistesleben und politische Tätigkeit", ebd., Bd 30 (1886), S. 149-52, 167-69), am 29.5.1849 in Paris angekommen (ders., .,Bekenntnisse eines Achtundvierzigers”. ebd., Bd 22 (1882), S. 131-34, 146-49). Durch einen ,wirklichen oder vermeintlichen Choleraanfall" behindert, mußte er Schütz, der von seiner Regierung durch mangelhafte Informationen und inkonsequente Handlungen in eine ..falsche Position" gebracht wurde. weitgehend die Initiative überlassen. Ruges Bedeutung war "gleich Null”. Marx. der als Bevollmächtigter des Zentralausschusses der Demokraten Deutschlands um den 2.6. in Paris ankam und dort mit der ,ganzen revolutionären Partei” zusammentraf, unterstützte trotz seiner Skepsis gegenüber der Politik der rheinpfälzischen und badischen Regierungen die Schütz-Blind-Mission nach Kräften (an Engels, 7.6., MEGA, a.a.O., S. 25f.). Aus späterer Sicht schrieben Marx und Engels, Blind habe das „Glück” gehabt, ,von einer reichlich grotesken Mission, mit der er von einer toten Regierung [Brentano] an eine ungeborene Regierung [Ledru-Rollin] beauftragt worden war, durch die beste- 
heimnißvollen Winken in engl. Blättern ${ }^{94}$ zu verstehn gegeben, daß er einen wichtigen diplomatischen Posten als Agent der deutschen ,republ. Regierung" zu Paris bekleidet hat!

In den ersten Jahren nach 1849 lebte er in Belgien ${ }^{95}$ mit seiner jetzigen Frau. Da diese Frau Kinder ${ }^{96}$ aus ihrer Ehe mit dem todten Banquier hat (denen Vermögen gehört) u. sie gleichzeitig Kinder mit Blind hat (damals noch nicht legitime), so erließ ein badischer Gerichtshof ein Urtheil, ${ }^{97}$ wonach die Kinder des Cohen der Wittwe Cohen abgenommen werden sollten wegen ihres „unmoralischen” Verhältnisses mit Ex-Studiosus Blind. D. Brüsseler Gericht gab diesem Urtheil Rechtskraft in Bel= /9/gien, u. dieß veranlaßte Blind mit d. Frau u. den Kindern nach England

hende französische Regierung [Louis Bonaparte] befreit zu werden, die ihn am 13. Juni bei der .friedlichen' Demonstration der erregten Pariser Nationalgarde verhaftete und schließlich des Landes verwies” (,Über Karl Blind”, a.a.O., S. 266). Für Einzeldaten der Schütz-Blind-Mission vgl. den Bericht des Augenzeugen S. Seiler, Das Complott vom 13. Juni 1849, oder der letzte Sieg der Bourgeoisie in Frankreich. Hamburg 1850. S. 33ff., 51 . ${ }^{94}$ "In June he was sent to Paris in the capacity of diplomatic envoy from the new Governments of Baden and Palatinate”, hatte Blind geschrieben. „M. Karl Blind”, a.a.O. 95 Blind, der zusammen mit Marx im Londoner Flüchtlingskomitee tätig war (,Aufruf zur Unterstützung deutscher Flüchtlinge", 20.9.1849, MEW, Bd 7, S. 545f.; Blind an „liebe Freundin” [Emma Herwegh?], 18.9., Stadtarchiv Mannheim, K1. Erw. 68, Nr 5; danach wollte er dem Komitee nur beitreten, um nicht als ,Handschuhdemokrat" bei den ,.Straubingern” zu erscheinen), hatte Marx gegenüber seinen Aufenthalt in Brüssel damit entschuldigt. daß die „Vermögensangelegenheiten” seiner Frau zu regeln seien (13.-14.10., in MEGA, a.a.O., S. 400); später bat er Marx sogar, gegen „Übelredende” ein ,politisches Mysterium" wegen seiner Abwesenheit aus London zu erfinden (1.-5.11.. ebd., S. 409). Ob Blind als Mitglied des Bundes der Kommunisten in Brüssel aktiv war, ist fraglich (Marx an Blind, 17.7.1850; C. Schramm an Marx, 9.9., ebd., S. 87, 632). 1852 ließ sich Blind. ., aus Belgien nothgedrungener Weise weggegangen", mit der Familie in London nieder (Blind an Marx, 16.11.1852, Marx-Engels-Nachlaß D 347, s. ferner Anm. 99).

96 Es handelt sich u.a. um Ferdinand Cohen-Blind, der nach seinem am 7.5.1866 auf Bismarck verübten Attentat im Gefängnis den Freitod wählte und für den Marx eine „besondere Sympathie” hatte, da er ein „alter Freund” seines verstorbenen Sohnes Edgar gewesen war (Engels an Marx, 9.5.1866; Marx an Engels, 10.5., MEW. Bd 31, S. 214f.), und um Mathilde Cohen-Blind, in den achtziger Jahren eine bekannte Übersetzerin, Schriftstellerin und Dichterin in England (The Poetical Works of Mathilde Blind, a.a.O.: ihr Nachlaß befand sich im Newnham College, Cambridge, wurde aber laut Auskunft vom 5.4.1982 verkauft). Für Blinds Reaktion auf das Attentat vgl. Der Deutsche Eidgenosse, 15.6.1866 und Mai 1867, sowie Rasch, „Deutsche Flüchtlinge in London". Über die Kinder aus zweiter Ehe ist wenig bekannt. Die Tochter Ottilie. verheiratete Hancock, vermachte den Nachlaß ihres Vaters dem British Museum.

97 Das badische Gericht hatte 1853 ,das ganze Vermögen der Kinder erster Ehe [...] sequestiert". Durch die Vermittlung von Marx wollte Blind den Rechtsbeistand von Ernest Jones erlangen (Marx an Blind, 26.9.1853, an Engels, 28.9., an Weydemeyer, Anfang Oktober, an Blind, 13.10., MEW, Bd 28, S. 593, 294, 594, 595, sowie Blind an Marx, 25.9., Marx-Engels-Nachlaß D 346). 
zu flüchten. Später ließ er in engl. Blättern drucken (u. hatte d. Unverschämtheit öffentlich bei einem Besuch in Süddeutschland zu renommiren $^{98}$ ), daß er aus politischen ${ }^{99}$ Gründen aus Belgien verjagt worden sei!

Seine nächste That werden Sie aus dem einliegenden Zettel sehn, den er in vielen Londoner Blättern hat drucken lassen. Anlaß gab die in einigen Blättern verbreitete Nachricht, Garibaldi habe sich für die Sklavenhal$t^{100}$ erklärt! Blind benutzte dieß um dem Garibaldi ein Brieflein zu erpressen, mit ,cordial greetings for Mrs Blind”. Was er dem Garibaldi vorgelogen hat, können Sie aus dessen Phrase sehn: „I thank you for your good news". ${ }^{101}$ Welche "good news" konnten in aller Welt im October 1864 mitgetheilt werden? Blind schrieb dem G. offenbar die ungeheuersten Lügen über d. Fortschritt des „Republikanismus” in Dtschld, welches wahrscheinlich nur auf B's Erscheinen wartet, um loszuschlagen.

Das Schönste an der Sache ist, daß das Londoner Blatt, woran Blind regelmäßiger Mitarbeiter, [10] das Organ der Schankwirthe (ganz passendes Lokal für einen „Blind”) fanatisch für die Conföderirten ist! Nebenbei bemerkt, jenes Blatt - The Morning Advertiser ${ }^{102}$ - ist $\mathrm{zu}$

98 ..Last year he returned for a short time to Baden. on the occasion of an unconditional amnesty being granted, and was enthusiastically received by all classes - the Second Chamber of Stuttgart entertaining him a grand banquet.” „M. Karl Blind”.

99 ..He afterwards lived in Belgium, but being threatened with expulsion when the authorities became aware of his presence, he found it necessary to return to Britain". Ebd. Wenn hier auch nicht ausdrücklich von politischen Gründen die Rede ist, so wird dieser Eindruck doch durch den Textzusammenhang erzeugt.

100 Der Index (Glasgow) hatte Garibaldis ..pro-Southern views" verbreitet, worauf der General antwortete: ,Non seulement j'en espère l'abolition de l'esclavage - mais je considère la question comme humanitaire - et malheur pour le Monde si le Nord ne sortait pas - victorieux de la lutte." (an Blind, 30.10.1864. Blind Papers. Vol. 2, f. 313) Blind, der Mitglied der 1862 gegründeten Emancipation Society in London war (s. Prospekt in der British Library), veröffentlichte die Richtigstellung in englischer Übersetzung (The Morning Advertiser, 9.11., S. 4: s. ferner Marx an Engels, 1.2.1865, MEW, Bd 31.S. 50).

101 Die Garibaldi-Zuschrift in The Morning Advertiser schloß: „I thank you for your good news. With cordial greatings to Mrs. Blind and to our friends." Die gute Nachricht über den Fortschritt des Republikanismus bezog sich wahrscheinlich auf Blinds Zeitschriftenprojekt. Der deutsche Eidgenosse sollte als Zeitschrift des Vereins „Deutsche Freiheit und Einheit" an die Stelle der bislang publizierten Flugschriften treten und von $\mathrm{N}$. Trübner in London und $\mathrm{O}$. Meißner in Hamburg herausgegeben werden. Die Vermittlung beim Hamburger Verlag übernahm K. Sieboldt; zur gleichen Zeit verhandelte W. Strohn in Marx' Auftrag mit O. Meißner über die Herausgabe des Kapitals (Marx an Engels, 1.2.1865; W. Strohn an Marx. 30.1., MEW, Bd 31. S. 49 und 620, Anm. 69). Garibaldi entwarf für den Eidgenossen - von Marx als „Lakai” betitelt (an Engels, 10.3., s. Anm. 83) - eine Zuschrift (Original in den Blind Papers, Vol. 4, ff. 149-51, in deutscher Ubersetzung abgedruckt in der genannten Zeitschrift, 15.7., S. 58).

102 Das 1794 gegründete Verlagsunternehmen war ein ..joint-stock venture of a large society of licensed victuallers. amongst whom subscription to the paper was the condition 
gleicher Zeit das Organ des Schnapses, der Low Church (des englischen Pietismus), des swell-mob, der sich mit Wetten, pricefights and so forth beschäftigt, u. der servilste Hund Palmerston's. From a literary standpoint, zählt es nicht mit in d. englischen Tagespresse, wird überhaupt nur in Taprooms gelesen.

So viel über den Mann.

Er hatte bei Lebzeiten Lassalle's gegen denselben eine Adresse ${ }^{103}$ erlassen, worin er selben verdächtigte. Doch hielt Lassalle nicht d. Mühe werth - so viel ich weiß - zu antworten. Nur wunderte mich, daß B. Becker, der vollständig mit d. Treiben Blind's bekannt ist, damals nicht die Gelegenheit benutzte um meine Enthüllungen über d. Blind an d. große Glocke zu hängen.

Einer der Gründe, warum ich der hiesigen Internationalen Arbeitergesellschaft beigetreten bin, ist, jenen Menschen zu enthüllen. ${ }^{104}$ [11] Nach meinen Erklärungen gegen Blind, worin ich ihn als ,infamen Lügner” u.

of membership". Sechzig Jahre lang hatte die Tageszeitung eine Auflage von 5000 Exemplaren, die fast ausschließlich in ,public-houses and coffee-houses” auslagen. The Encyclopaedia Britannica, 11. Aufl., New York 1911, Bd 19, S. 559a.

${ }^{103}$ K. Blind, ..Ein Freundeswort an Deutschlands Arbeiter, Bürger und Bauern", in: Hermann, 6.6.1863, dann Neue Frankfurter Zeitung, 8.6., Der Beobachter, 9.6., Frankfurter Reform, 10.6., s. auch FL-ADAV, S. 190, C 153; daneben erschien das Freundeswort als Flugblatt von Blinds Verein. Unter Hinweis auf Lassalles Schriften Was nun? und Offenes Antwortschreiben sieht Blind durch die Arbeiteragitation und durch die Kampagne für die Oktroyierung des allgemeinen Stimmrechts ,.die ersten Grundsätze der deutschen Demokratie" umgestoßen. Für Reaktionen darauf vgl. Anm. 73.

10-4 Der hier von Marx genannte Grund erweitert die Erkenntnisse, die bisher in der Literatur zur Geschichte der IAA (s. neuerdings Die Erste Internationale, 1864-1870, hrsg. von I. A. Bach u.a., Bd l. Moskau 1981) für die Gründungsphase und für Marx Beitritt gemacht worden sind. „Jenen Menschen zu enthüllen”. hieß, Blinds selbstgefällige Betriebsamkeit bei der Verfolgung republikanischer und internationalistischer Ziele in einer Situation bloßzustellen, in der ,wirkliche Mächte" von wiederauflebenden französischen und englischen Arbeiterbewegungen (s. Anm. 76) am Werk waren und die Gefahr bestand, diese „Mächte” durch klassenübergreifende Republikanismus- und Nationalismus-Parolen à la Mazzini, Blind, Ledru-Rollin u.a. zu desorientieren. Marx hatte sich nach langer politischer Zurückgezogenheit entschlossen, die von Engels und von ihm seit 1848 entwickelte „Ansicht” als Repräsentant der deutschen Arbeiter in einer internationalen Arbeiterassoziation zur Wirkung zu bringen. Daher konnte er sich auch in der Nachfolgefrage für Lassalle, wo es sich um die Präsidentschaft in einem Nationalen Arbeiterverein handelte, distanziert verhalten (s. Anm. 15), während er sich bei der Diskussion über die zu entwerfenden programmatischen Dokumente der IAA energisch einschaltete und besonders mazzinistische Auffassungen in der Arbeiter- und Nationalitätenfrage bekämpfte (an Engels, 4.11.1864, a.a.O., S. 10-16). Später gründete Mazzini ein „International Republican Committee”, an dem u.a. Blind, Kinkel und Ledru-Rollin teilnahmen (Marx an Engels, 13.5.1866, MEW, Bd 31, S. 219). 
„Fälscher v. Zeugnissen” gebrandmarkt habe, kann ich mich natürlich nicht mehr in Polemik mit dem Subjekt einlassen. Ich behalte mir vor ihn zu gelegner Zeit als komische Figur zu behandeln. Wenn ich jetzt seinen mir übrigens unbekannten Wisch gegen Lassalle zum Grund eines Angriffs mache, wird man hier, wo man meine Todtfeindschaft gegen den B. kennt, glauben, daß ich in der That Lassalle's Namen nur zum Vorwand meiner Privatrache mache.

Schicken Sie mir übrigens die (ged ruckte) Erklärung der Arbeiter zu. Ich werde dafür sorgen, daß sie in ein hiesiges deutsches Blatt kommt u. sollte Stud. Blind dann weitere Schritte thun, so giebt er mir vielleicht die Gelegenheit über ihn herzufallen. ${ }^{105}$

Jedenfalls verlassen Sie sich darauf, daß ihm nichts geschenkt wird. Einstweilen können Sie gar nichts beßres thun als [12] meine gerichtlichen

105 Diese Gelegenheit ergab sich für Marx ..glücklicherweise" (an S. v. Hatzfeldt. 26.11.1864, MEW. Bd 31, S. 426) schon wenige Tage später. Von nicht bekannter Seite waren ihm zwei Artikel des Beobachters zugesandt worden. Beim ersten. .,Bescheidenheit - ein Ehrenkleid" (21.10.: ein Exemplar mit anstreichungen - von Marx? - im MarxEngels-Nachlaß, Mappe ,Knipsels Marx-Engels- en Jung-archief”) hatte der Redakteur A. Mayer ein Blind-Rundschreiben des Vereins „Deutsche Freiheit und Einheit” vom 12.9. betr. die amerikanische Präsidentenwahl kritisiert. Mayer warf Blind ..Selbstverblendung" und "Eitelkeit" - was schon zur Vogt-Affäre und zu Marx' Pamphlet geführt habe - sowie .Diktatur in Spe" vor. Blind ließ darauf von Bronner (s. Anm. 74) eine Antwort schreiben. in der die Vogt-Affäre als .,abgethaner Streit" und Blinds seit ..bald zwölf Jahren" währende Tätigkeit "für 6-7 Millionen Deutsche” in Amerika hervorgehoben wurde (.,Karl Blind”, ebd., 17.11.; der erste Teil des Artikels besteht aus einer redaktionellen Einleitung. in der die Vorwürfe gegen Blind halbwegs zurückgenommen wurden, der zweite Teil aus der Korrespondenz „Bradford, 25. Oct."). Zur gleichen Zeit hatte Weydemeyer von St Louis aus auf Blinds ..Harlekinaden” in der amerikanischen Öffentlichkeit, die ausschließlich mit dem Bürgerkrieg beschäftigt war, hingewiesen (s. Anm. 37). Marx setzte den dreifachen Anstoß - durch die Gräfin, die beiden Beobachter-Artikel und durch Weydemeyer - in einen konkreten Plan um (an Engels, 25.11., an S. v. Hatzfeldt, 26.11., an Weydemeyer, 29.11., a.a.O., S. 31 f., 426, 428f.) und verfaßte die Blind-Erklärung, ,an den Redakteur des Beobachters, London, 28. Nov. 1864". Sie wurde an verschiedene deutsche Zeitungen versandt. erschien aber zu Marx' Verdruß nur in K. v. Bruhns Zeitung, dazu noch mit der Notiz: „Dieser Aufsatz ist uns durch eine zweite Hand [S. v. Hatzfeldt] zugegangen und nur wegen besonderer Berücksichtigung derselben findet jener eine Aufnahme im Nordstern" (10.12., S. 2-3, MEW, Bd 16. S. 22-24; ferner die Beschwerde von Marx im Brief an S. v. Hatzfeldt, 22.12., MEW, Bd 31 , S. 434; die Gräfin hatte außerdem den Mazzini-Passus, in dem Marx Blind einen ..grotesken Mazzini-Scapin" nennt, in ,grotesken Clown” umgewandelt. s. Liebknecht an Marx, 2.12., Marx-Engels-Nachlaß D 3096). Damit war für Marx diese Blind-Episode beendet; er hatte aus .,alter Freundschaft" zu Lassalle für die Gräfin die ..gewünschte Erklärung” gegen Blinds ,feige Frechheit” abgegeben, ohne sich „,mit den mißfälligen Seiten der Lassalleschen Agitation zu identifizieren" (an Weydemeyer, a.a.O.. an Kugelmann, 23.2.1865, MEW, Bd 31, S. 451). Blind replizierte noch einmal spöttisch, nachdem Marx und Engels sich vom Social-Demokraten losgesagt hatten (Fragment. Blind Papers, Vol. 4, ff. 108-09; Neue Frankfurter Zeitung. 5.3.1865. S. I). 
Enthüllungen über Blind als ,,infamen Lügner” u. „Fälscher” möglichst in Dtschld verbreiten. (Wenn sein Wisch über Lassalle hier einen Hund vom Ofen gelockt hätte, wäre mir jedenfalls ein od. das andre Wort darüber zu Ohren gekommen.) Dieß würde ihn zwingen sein Schweigen zu brechen u. mich so befähigen meinen Mund wieder zu öffnen über ein Subjekt, von dem ich öffentlich erklärt habe, daß er infam ist.

Meine Reise nach Berlin wäre, von allem andern abgesehn, völlig unmöglich gewesen in Folge des Wiederausbruchs der schändlichen Carbunkelkrankheit, an der ich mit geringen Unterbrechungen seit 14 Monaten laborire.

Uebrigens können Sie sicher sein, daß ich die mir passende Gelegenheit [Sie müssen mir aber überlassen den mir günstig scheinenden Zeitpunkt zu wählen] ergreifen werde um meinen zu früh geraubten Freund vor allen böslichen Angriffen zu schützen.

Ihr ganz ergebener K.M.

\section{EXKURS}

Der „Republikanische Protest” war zuerst in der Neuen Frankfurter Zeitung (s. Anm. 35), dann - um Auszüge aus Lassalles Verteidigungsrede im Berliner Hochverratsprozeß vom 12. März 1864 erweitert $^{106}$ - im Hermann, 8. Oktober, S. 1, erschienen; für eine dritte Variante s. Anm. 37. Außerdem kam der Protest in der Reihe des Vereins „Deutsche Freiheit und Einheit" (s. Anm. 69) in London heraus.

Blind wandte sich als Repräsentant in England lebender, deutscher Republikaner gegen die von Genf her aufgestellte Behauptung, ,die republikanische Partei habe einen ihrer besten Vertreter an Lassalle verloren!" Lassalles Ansicht über die Oktroyierung des allgemeinen Stimmrechts hätte einen mit ,schein-socialistischen Phrasen verkleideten Napoleonismus" erkennen lassen. Der Protest polemisierte gegen einen von Friedrich Reusche in Verbindung mit der Gräfin verfaßten Genfer Aufruf. ${ }^{107}$ Der als Flugblatt - ein Blatt, eine Seite mit deutschem, die an-

106 Gesammelte Reden und Schriften, Bd 4, S. 153-55, 159-60.

${ }_{107}$ FL-ADAV, S. 182, C 99, allerdings J. Ph. Becker zugeschrieben; Original ADAV/H 192, mit einem Entwurf von der Hand der Gräfin; wegen der Verfasserschaft vgl. F. Reusches gezeichneten Artikel „Der Kampf der Mittelmäßigkeiten gegen das Genie” im Nordstern, 26.11., S. 2-3. Zur umstrittenen Person Reusche, den J. Ph. Becker einmal „Generalfeldzeugmeister aller Lüge” (W. Rüstow an Becker, 26.1.1865; Becker an S. v. Hatzfeldt, 8.9. 1864, ADAV/H 6/1) genannt hatte, s. Offermann, Arbeiterbewegung und liberales Bürgertum, a.a.O., S. 459f., Anm. 62. Die diversen Materialien im Becker- 
dere mit französischem Text - verbreitete Aufruf bezeichnete Lassalle als die „Hoffnung des Vaterlandes und der deutschen Republikaner" (bzw. „l'espoir de son pays et du parti social démocratique, dont il était le chef'), der Opfer geworden sei der ,schmählichsten Intrigue, die jemals von verworfenen Personen mit einem edeln, großen Mann gespielt wurde" (bzw. „tombé victime de la plus horrible trahison, de l'intrigue la plus infâme"), und lud die Genfer Republikaner aller Nationen zur Leichenfeier im Temple Unique am 2. September im Namen des „Comités der deutschen Republikaner” ein. Das Flugblatt wurde nachgedruckt im Nordstern, 10. September, S. 2 (,Trauerfeier in Genf”) im Anschluß an die von J. Ph. Becker u.a. erlassene „Proklamation”. ${ }^{108}$

Der erste Angriff gegen den Genfer Aufruf kam von seiten der Neuen Frankfurter Zeitung, die seit Leopold Sonnemanns Streit mit Lassalle im Mai 1863 diesen als ,falschen Demokraten” verfolgt hatte. ${ }^{109}$ In dem Artikel „Lassalle's Ende” (7. September, S. 2-3), verfaßt nach Mitteilungen „genauer Freunde von Lassalle" (11. September, S. 1), wurde der Aufruf als erster Beweis dafür gewertet, den ,mysteriösen” Duellvorfall in ,politischer Hinsicht auszubeuten und Lassalle zum Martyrer für seine ,Arbeiterbataillone' zu stempeln". Repliken auf die Artikel der Neuen Frankfurter Zeitung erfolgten von J. Ph. Becker und G. Herwegh. ${ }^{110}$

Nachlaß und in der Sammlung ADAV/H geben Auskunft über Reusches Umtriebigkeit, vgl. ferner FL-ADAV, S. 265, C 741-42. Ende 1864, als Reusche gegen Blind vorging (vgl. Anm. 36), setzte er sich auch mit Marx in Verbindung (Reusche an Becker, ca Dezember, Becker-Nachlaß D II 769; Jenny Marx an Engels, 30.3.1865, ferner Engels an Marx, 16.4., MEW, Bd 31, S. 584, 108).

108 FL-ADAV, S. 183, C 99a. Kurz vorher war es in das ADAV-Zirkular, 9.9., eingegangen und bildete den ersten Erklärungsversuch für die ahnungslosen Vereinsmitglieder (ADAV/H 162).

109 H. Ebeling, Der Kampf der Frankfurter Zeitung gegen Ferdinand Lassalle und die Gründung einer selbständigen Arbeiterpartei, Leipzig 1931.

${ }^{110}$ S. Anm. 14: Emma Herwegh an [Ludwig Schweigert], 14.9., in Ferdinand Lassalle's Briefe an Georg Herwegh, a.a.O., S. 104. Für Blinds Beziehung zur Neuen Frankfurter Zeitung s. Anm. 103. 